\title{
Enantioselective Synthesis of Cyclic Secondary Amines through Mo- Catalyzed Asymmetric Ring-Closing Metathesis (ARCM)
}

\author{
Sarah J. Dolman, ${ }^{1}$ Richard R. Schrock, ${ }^{*},{ }^{1}$ and Amir H. Hoveyda*, ${ }^{2}$ \\ ${ }^{1}$ Department of Chemistry, Massachusetts Institute of Technology, \\ Cambridge, Massachusetts 02139 \\ ${ }^{2}$ Department of Chemistry, Merkert Chemistry Center, Boston College \\ Chestnut Hill, Massachusetts 02467
}

\section{SUPPORTING INFORMATION}

\section{GENERAL}

Infrared (IR) spectra were recorded on ThermoNicolet Avatar 360 spectrophotometer, $v_{\max }$ in $\mathrm{cm}^{-1}$. Bands were characterized as broad (br), strong (s), medium (m), and weak (w). ${ }^{1}$ H NMR spectra were recorded on Varian VXR $500(500 \mathrm{MHz})$ or Unity 300 (300 $\mathrm{MHz}$ ) spectrophotometers. Chemical shifts are reported in ppm from tetramethylsilane with the solvent resonance as the internal standard $\left(\mathrm{C}_{6} \mathrm{D}_{6}: \delta 7.16, \mathrm{CDCl}_{3}: \delta\right.$ 7.26). Data are reported as follows: chemical shift, multiplicity ( $\mathrm{s}=$ singlet, $\mathrm{d}=$ doublet, $\mathrm{t}=$ triplet, $\mathrm{q}$ $=$ quartet, $\mathrm{br}=$ broad, $\mathrm{m}=$ multiplet $)$, coupling constants $(\mathrm{Hz})$, integration, and assignment. ${ }^{13} \mathrm{C}$ NMR spectra were recorded on Varian VXR $500(125 \mathrm{MHz})$ spectrophotometer with complete proton decoupling. Chemical shifts are reported in ppm from tetramethylsilane with the solvent resonance as the internal standard $\left(\mathrm{C}_{6} \mathrm{D}_{6}\right.$ : $\delta 128.39, \mathrm{CDCl}_{3}: \delta$ 77.7). Enantiomer ratios were determined by chiral HPLC (Chiral Technologies Chiralpak AS (4.6 $\mathrm{mm} \theta \times 250 \mathrm{~mm})$, Chiralcel OJ $(4.6 \mathrm{~mm} \theta \times 250 \mathrm{~mm})$ or Chiralcel OD $(4.6 \mathrm{~mm} \theta \times 250 \mathrm{~mm}))$ in comparison with authentic racemic materials/ High-resolution mass spectrometry was performed at the Massachusetts Institute of Technology, Department of Chemistry Instrumentation Facility (Cambridge, MA).

All reactions were conduction in oven $\left(135{ }^{\circ} \mathrm{C}\right)$ or flame-dried glassware under an inert atmosphere of dry $\mathrm{N}_{2}$. All metathesis substrates were dried by storing over molecular sieves under a $\mathrm{N}_{2}$ atmosphere for a minimum of 12 hours prior to use. Handling of all Mo catalysts was performed in a drybox. $\mathrm{Et}_{2} \mathrm{O}$, toluene, and pentane were sparged with $\mathrm{N}_{2}$ and then passed through an activated alumina column or distilled from sodium/benzophenone ketyl. Benzyl potassium was prepared by the literature method. All reagents were used as received from Aldrich Chemical Co., Lancaster Synthesis, or Strem Chemicals, Inc. unless otherwise stated. Mo complexes 3, 4, 5 and 9 were prepared according to published procedures.

\section{EXPERIMENTAls FOR PRECURSORS}

Pent-4-enoic acid (4-methoxy-phenyl)-amide: To a solution of p-anisidine, (10 g, 81

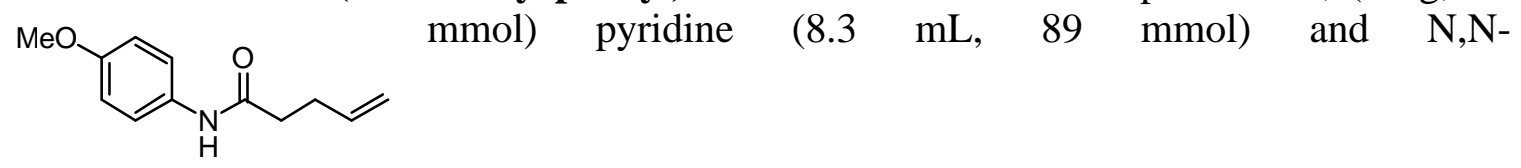


dimethylaminopyridine $(500 \mathrm{mg}, 4 \mathrm{mmol})$ in dichloromethane at $0{ }^{\circ} \mathrm{C}$ was added dropwise 4-pentenoyl chloride $(9.9 \mathrm{~mL}, 89 \mathrm{mmol})$. After warming to room temperature, $10 \%$ $\mathrm{HCl}$ in water $(250 \mathrm{~mL})$ was added to the solution. This mixture was extracted three times with dichloromethane $(500 \mathrm{~mL})$. All organic extracts were combined, washed with saturated $\mathrm{NaHCO}_{3}$ solution, then $\mathrm{NaCl}$ solution, dried over $\mathrm{MgSO}_{4}$ and concentrated in vacuo to a brown solid. The solid was recrystallized from a solution of ether and pentane to afford a white powder, $12.2 \mathrm{~g}, 59 \mathrm{mmol}, 73 \%$ yield. ${ }^{1} \mathrm{H}$ NMR $\left(500 \mathrm{MHz}, \mathrm{C}_{6} \mathrm{D}_{6}\right) \delta 7.52$ $(\mathrm{d}, 2 \mathrm{H}, \mathrm{J}=9 \mathrm{~Hz}$, aryl $\mathrm{CH}) 6.76(\mathrm{~d}, 2 \mathrm{H}, \mathrm{J}=9 \mathrm{~Hz}$, aryl $\mathrm{CH}) 5.74\left(\mathrm{~m}, 1 \mathrm{H}, \mathrm{CH}=\mathrm{CH}_{2}\right) 4.97$ $\left(\mathrm{m}, 2 \mathrm{H}, \mathrm{CH}=\mathrm{CH}_{2}\right) 3.27\left(\mathrm{~s}, 3 \mathrm{H}, \mathrm{CH}_{3} \mathrm{O}\right) 2.36\left(\mathrm{~m}, 2 \mathrm{H}, \mathrm{C}(\mathrm{O}) \mathrm{CH}_{2} \mathrm{CH}_{2}\right) 1.97(\mathrm{t}, 2 \mathrm{H}, \mathrm{J}=7.5$ $\left.\mathrm{Hz}, \mathrm{C}(\mathrm{O}) \mathrm{CH}_{2} \mathrm{CH}_{2}\right) .{ }^{13} \mathrm{C} \mathrm{NMR}\left(125 \mathrm{MHz}, \mathrm{C}_{6} \mathrm{D}_{6}\right): \delta 170.3157 .0138 .0132 .5122 .1115 .8$ 114.6 55.3 55.2 36.8 30.2. IR (KBr): 3308.5 (s) 3082.0 (w) $2952.4(\mathrm{~m}) 1875.3(\mathrm{w}) 1652.9$ (s) 1601.7 (m) 1542.1 (s) 1515.7 (s) 1410.3 (s) 1247.2 (s) 1173.5 (m) 1030.3 (s). HRMS (ESI) Calcd for $\mathrm{C}_{12} \mathrm{H}_{15} \mathrm{NO}_{2}[\mathrm{M}+\mathrm{Na}]$ : 228.0995. Found: 228.0999 .

Pent-4-enoic acid (4-bromo-phenyl)-amide: Analogous preparation as above: $8.45 \mathrm{~g}$,

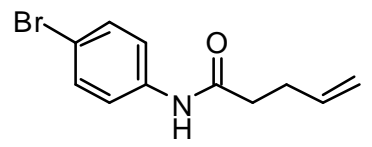
$40 \mathrm{mmol}, 98 \%$ yield. ${ }^{1} \mathrm{H} \mathrm{NMR}\left(500 \mathrm{MHz}, \mathrm{CD}_{2} \mathrm{Cl}_{2}\right): \delta 7.86(\mathrm{br}$, $1 \mathrm{H}, \mathrm{NH}) 7.41\left(\mathrm{~m}, 4 \mathrm{H}\right.$, aryl CH) $5.86\left(\mathrm{~m}, 1 \mathrm{H}, \mathrm{CH}=\mathrm{CH}_{2}\right) 5.09(\mathrm{dd}$, $\left.1 \mathrm{H}, \mathrm{J}=17.1 \mathrm{~Hz}, 0.9 \mathrm{~Hz}, \mathrm{CH}=\mathrm{CH}_{\mathrm{a}} \mathrm{H}_{\mathrm{b}}\right) 5.02(\mathrm{dd}, 1 \mathrm{H}, \mathrm{J}=10.7 \mathrm{~Hz}$, $\left.0.9 \mathrm{~Hz}, \mathrm{CH}=\mathrm{CH}_{\mathrm{a}} \mathbf{H}_{\mathbf{b}}\right) 2.43\left(\mathrm{~s}, 4 \mathrm{H}, \mathrm{CH}_{\mathbf{2}} \mathrm{CH}_{2}\right) .{ }^{13} \mathrm{CNMR}(125 \mathrm{MHz}$, $\mathrm{CD}_{2} \mathrm{Cl}_{2}$ ): $\delta 171.5137 .9137 .8132 .3122 .0116 .9116 .037 .1$ 29.8. IR (KBr): 3285.3 (s) $2978.9(\mathrm{w}) 1655.5(\mathrm{~s}) 1589.1(\mathrm{~m}) 1529.6(\mathrm{~s}) 1488.2(\mathrm{~s}) 1395.1(\mathrm{~m}) 1298.6(\mathrm{w}) 1188.9(\mathrm{w})$ 1072.3 (m). HRMS (ESI') Calcd for $\mathrm{C}_{11} \mathrm{H}_{12} \mathrm{NOBr}[\mathrm{M}+\mathrm{Na}]: 275.9994$. Found: 275.9997.

Pent-4-enoic acid (3-triflouromethyl-phenyl)-amide: Analogous preparation as above:<smiles>C=CCCC(=O)Nc1cccc(C(F)(F)F)c1</smiles>
$10 \mathrm{~g}, 41 \mathrm{mmol}, 100 \%$ yield. ${ }^{1} \mathrm{H} \mathrm{NMR}\left(500 \mathrm{MHz}, \mathrm{C}_{6} \mathrm{D}_{6}\right): \delta 7.87(\mathrm{~d}$, $1 \mathrm{H}, \mathrm{J}=8.2 \mathrm{~Hz}$, aryl CH) $7.33(\mathrm{br}, 1 \mathrm{H}$, aryl CH) $7.05(\mathrm{~d}, 1 \mathrm{H}, \mathrm{J}=7.9$ $\mathrm{Hz}$, aryl CH) $6.89(\mathrm{t}, 1 \mathrm{H}, \mathrm{J}=7.9 \mathrm{~Hz}$, aryl $\mathbf{C H}) 6.23(\mathrm{br}, 1 \mathrm{H}, \mathbf{N H})$ $5.71\left(\mathrm{~m}, 1 \mathrm{H}, \mathrm{CH}=\mathrm{CH}_{2}\right) 4.98\left(\mathrm{~m}, 1 \mathrm{H}, \mathrm{CH}=\mathrm{CH}_{\mathrm{a}} \mathrm{H}_{\mathrm{b}}\right) 4.95(\mathrm{~m} 1 \mathrm{H}$, $\left.\mathrm{CH}=\mathrm{CH}_{\mathrm{a}} \mathbf{H}_{\mathbf{b}}\right) 2.29\left(\mathrm{~m}, 2 \mathrm{H}, \mathrm{CH}_{2}\right) 1.83\left(\mathrm{~m}, 2 \mathrm{H}, \mathrm{CH}_{2}\right) .{ }^{13} \mathrm{C} \mathrm{NMR}\left(125 \mathrm{MHz}, \mathrm{C}_{6} \mathrm{D}_{6}\right): \delta 139.5$ 137.5130 .1127 .1123 .3120 .8116 .6116 .136 .8 29.8. IR (KBr): 3302.3 (s) $2981.9(\mathrm{~m})$ 1667.0 (s) 1558.2 (s) 1493.7 (s) 1447.6 (s) 1334.6 (s) 1282.0 (m) 1166.9 (s) 1071.1 (s). HRMS (ESI) Calcd for $\mathrm{C}_{12} \mathrm{H}_{12} \mathrm{NOF}_{3}[\mathrm{M}+\mathrm{Na}]: 266.0763$. Found: 266.0762.

Pent-4-enoic acid (2-methoxy-phenyl)-amide: Analogous preparation as above: $8.2 \mathrm{~g}$,<smiles>C=CCCC(=O)Nc1ccccc1OC</smiles>
$40 \mathrm{mmol}, 98 \%$ yield. ${ }^{1} \mathrm{H}$ NMR $\left(500 \mathrm{MHz}, \mathrm{CD}_{2} \mathrm{Cl}_{2}\right): \delta 8.31(\mathrm{~d}, 1 \mathrm{H}, \mathrm{J}$ $=7.9 \mathrm{~Hz}$, aryl CH) $7.79(\mathrm{br}, 1 \mathrm{H}, \mathrm{NH}) 7.02(\mathrm{dt}, 1 \mathrm{H}, \mathrm{J}=7.9 \mathrm{~Hz}, \mathrm{~J}=1.2$ $\mathrm{Hz}$, aryl CH) $6.92(\mathrm{dd}, 1 \mathrm{H}, \mathrm{J}=7.9 \mathrm{~Hz}, 1.2 \mathrm{~Hz}$, aryl CH) $6.89(\mathrm{dd}, 1 \mathrm{H}$, $\mathrm{J}=8.2 \mathrm{~Hz}, \mathrm{~J}=1.2 \mathrm{~Hz}$, aryl $\mathrm{CH}) 5.88\left(\mathrm{~m}, 1 \mathrm{H}, \mathrm{CH}=\mathrm{CH}_{2}\right) 5.10(\mathrm{~d}, 1 \mathrm{H}$, $\left.\mathrm{J}=17.1 \mathrm{~Hz}, \mathrm{CH}=\mathrm{CH}_{\mathrm{a}} \mathrm{H}_{\mathrm{b}}\right) 5.02\left(\mathrm{~d}, 1 \mathrm{H}, \mathrm{J}=10.7 \mathrm{~Hz}, \mathrm{CH}=\mathrm{CH}_{\mathrm{a}} \mathbf{H}_{\mathbf{b}}\right) 3.86\left(\mathrm{~s}, 3 \mathrm{H}, \mathrm{CH}_{3} \mathrm{O}\right) 2.45$ $\left(\mathrm{m}, 2 \mathrm{H}, \mathrm{CH}_{2}\right) .{ }^{13} \mathrm{C}$ NMR (125 MHz, $\left.\mathrm{CD}_{2} \mathrm{Cl}_{2}\right): \delta 170.7137 .7128 .4123 .9121 .3120 .0$ 115.8 110.5 56.2 37.5 29.9. IR (KBr): 3324.8 (s) 2938.8 (m) 1678.9 (s) 1600.4 (s) 1524.0 (s) 1459.6 (s) 1434.1 (w) 1355.5 (s) 1253.4 (m) 1175.6 (m) 1047.3 (m). HRMS (ESI') Calcd for $\mathrm{C}_{12} \mathrm{H}_{15} \mathrm{NO}_{2}[\mathrm{M}+\mathrm{Na}$ ]: 228.0995. Found: 228.0996 .

Pent-4-enoic acid (2-bromo-phenyl)-amide: Analogous preparation as above: $6.1 \mathrm{~g}, 24$<smiles>C=CCCC(=O)Nc1ccccc1Br</smiles>
mmol, $70 \%$ yield. ${ }^{1} \mathrm{H}$ NMR $\left(500 \mathrm{MHz}, \mathrm{CD}_{2} \mathrm{Cl}_{2}\right): \delta 8.29(\mathrm{~d}, 1 \mathrm{H}, \mathrm{J}=$ 
$7.9 \mathrm{~Hz}$, aryl CH) $7.66(\mathrm{br}, 1 \mathrm{H}, \mathrm{NH}) 7.55(\mathrm{dd}, 1 \mathrm{H}, \mathrm{J}=7.9 \mathrm{~Hz}, \mathrm{~J}=1.5 \mathrm{~Hz}$, aryl CH) 7.31 (dt, $1 \mathrm{H}, \mathrm{J}=7.8 \mathrm{~Hz}, 1.2 \mathrm{~Hz}$, aryl CH) $6.99(\mathrm{dt}, 1 \mathrm{H}, \mathrm{J}=7.8 \mathrm{~Hz}, \mathrm{~J}=1.5 \mathrm{~Hz}$, aryl CH) 5.91 $\left(\mathrm{m}, 1 \mathrm{H}, \mathrm{CH}=\mathrm{CH}_{2}\right) 5.14\left(\mathrm{~d}, 1 \mathrm{H}, \mathrm{J}=17.1 \mathrm{~Hz}, \mathrm{CH}=\mathrm{CH}_{\mathbf{a}} \mathrm{H}_{\mathrm{b}}\right) 5.06(\mathrm{~d}, 1 \mathrm{H}, \mathrm{J}=10.1 \mathrm{~Hz}$, $\left.\mathrm{CH}=\mathrm{CH}_{\mathrm{a}} \mathbf{H}_{\mathbf{b}}\right) 2.51\left(\mathrm{~m}, 2 \mathrm{H}, \mathbf{C H}_{2}\right) .{ }^{13} \mathrm{C}$ NMR $\left(125 \mathrm{MHz}, \mathrm{CD}_{2} \mathrm{Cl}_{2}\right): \delta 170.9137 .4136 .4$ 132.8128 .7125 .6122 .7116 .1114 .037 .4 29.8. IR (KBr): 3273.7 (s) 1659.8 (s) 1579.6 (m) $1526.9(\mathrm{~s}) 1438.3(\mathrm{~m}) 1414.3(\mathrm{~m}) 1374.5(\mathrm{w}) 1291.7(\mathrm{~m}) 1186.3(\mathrm{~m}) 1027.3(\mathrm{~m})$. HRMS (ESI) Calcd for $\mathrm{C}_{11} \mathrm{H}_{12} \mathrm{NOBr}$ [M + Na]: 275.9994. Found: 275.9992.

2-Vinyl-phenylamine: 2-Amino-phenethylalcohol (18 g, $131 \mathrm{mmol})$ and potassium

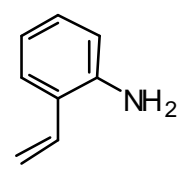
hydroxide $(7.36 \mathrm{~g}, 131 \mathrm{mmol})$ were combined in a distillation apparatus, then heated to $180{ }^{\circ} \mathrm{C}$ under vacuum. A clear, colorless oil was distilled over 4 hours at $55-60{ }^{\circ} \mathrm{C}$ at 0.25 Torr, $12.1 \mathrm{~g} 102 \mathrm{mmol}, 78 \%$ yield. ${ }^{1} \mathrm{H}$ NMR $\left(500 \mathrm{MHz}, \mathrm{C}_{6} \mathrm{D}_{6}\right): \delta 7.27(\mathrm{dd}, 1 \mathrm{H}, \mathrm{J}=1.5 \mathrm{~Hz}, 7.6 \mathrm{~Hz}$, aryl CH) $6.99(\mathrm{dt}, 1 \mathrm{H}$, $\mathrm{J}=1.5 \mathrm{~Hz}, 7.3 \mathrm{~Hz}$, aryl CH) $6.71(\mathrm{dt}, 1 \mathrm{H}, \mathrm{J}=1.2 \mathrm{~Hz}, 7.3 \mathrm{~Hz}$, aryl CH) $6.57(\mathrm{dd}, 1 \mathrm{H}, \mathrm{J}=$ $\left.11.2 \mathrm{~Hz}, 17.4 \mathrm{~Hz}, \mathrm{ArCH}=\mathrm{CH}_{2}\right) 6.31(\mathrm{dd}, 1 \mathrm{H}, \mathrm{J}=0.9 \mathrm{~Hz}, 7.9 \mathrm{~Hz}$, aryl CH) $5.50(\mathrm{dd}, 1 \mathrm{H}, \mathrm{J}$ $\left.=1.5 \mathrm{~Hz}, 17.4 \mathrm{~Hz}, \mathrm{CH}=\mathrm{CH}_{\mathbf{a}} \mathrm{H}_{\mathrm{b}}\right) 5.09\left(\mathrm{dd}, 1 \mathrm{H}, \mathrm{J}=1.5 \mathrm{~Hz}, 11.2 \mathrm{~Hz}, \mathrm{CH}=\mathrm{CH}_{\mathrm{a}} \mathbf{H}_{\mathbf{b}}\right) 3.00(\mathrm{br}$, $2 \mathrm{H}, \mathrm{NH}_{2}$ ). This spectrum matches published material ${ }^{1-3}$ on this compound, therefore no further spectral data were obtained.

N-(2-Vinyl-phenyl)-formidic acid ethyl ester: 2-Vinyl-phenylamine, (2.15 g, $18 \mathrm{mmol})$<smiles>C=Cc1ccccc1/N=C/O</smiles>
triethyl orthoformate $(3.9 \mathrm{~mL}, 23 \mathrm{mmol})$ and $p$-toluenesulfonic acid $(20$ $\mathrm{mg}, 90 \mu \mathrm{mol})$ were heated to $130{ }^{\circ} \mathrm{C}$ to remove 2 equivalents of ethanol in a Dean-Stark condenser. After the mixture was cooled, residual orthoester was removed at room temperature under Schlenk vacuum.

Subsequently, the crude material was distilled at reduced pressure with heating to afford $2.16 \mathrm{~g}(12 \mathrm{mmol})$ of a clear, colorless oil: $68 \%$ yield. ${ }^{1} \mathrm{H}$ NMR $\left(500 \mathrm{MHz}, \mathrm{C}_{6} \mathrm{D}_{6}\right) \delta 7.52$ $(\mathrm{dd}, 1 \mathrm{H}, \mathrm{J}=1.5 \mathrm{~Hz}, 7.6 \mathrm{~Hz}$, aryl CH) $7.37(\mathrm{dd}, 1 \mathrm{H}, \mathrm{J}=11.0 \mathrm{~Hz}, 17.7 \mathrm{~Hz}$, aryl CH) 7.27 $(\mathrm{s}, 1 \mathrm{H}, \mathrm{N}=\mathrm{CH}) 6.99\left(\mathrm{~m}, 2 \mathrm{H}\right.$, aryl $\left.\mathrm{CH}+\mathrm{CH}=\mathrm{CH}_{2}\right) 6.57(\mathrm{dd}, 1 \mathrm{H}, \mathrm{J}=1.5 \mathrm{~Hz}, 7.6 \mathrm{~Hz}$, aryl CH) $5.71\left(\mathrm{dd}, 1 \mathrm{H}, \mathrm{J}=17.7 \mathrm{~Hz}, 1.4 \mathrm{~Hz}, \mathrm{CH}=\mathrm{CH}_{\text {cis }} \mathrm{H}_{\text {trans }}\right) 5.22(\mathrm{dd}, 1 \mathrm{H}, \mathrm{J}=11.1 \mathrm{~Hz}, 1.4$ $\left.\mathrm{Hz}, \mathrm{CH}=\mathrm{CH}_{\mathrm{cis}} \mathrm{H}_{\text {trans }}\right) 4.11\left(\mathrm{q}, 2 \mathrm{H}, \mathrm{J}=7.0 \mathrm{~Hz}, \mathrm{OCH}_{2} \mathrm{CH}_{3}\right) 1.06(\mathrm{t}, 3 \mathrm{H}, \mathrm{J}=7.0 \mathrm{~Hz}$, $\left.\mathrm{OCH}_{2} \mathrm{CH}_{3}\right) .{ }^{13} \mathrm{C}$ NMR $\left(125 \mathrm{MHz}, \mathrm{C}_{6} \mathrm{D}_{6}\right) \delta 154.8146 .6134 .4131 .8129 .2126 .2125 .0$ 120.9114 .1 62.6 14.6. IR (NEAT, NaCl plates): 3064.8 (m) 2931.5 (s) 1646.3 (s) 1594.3 (s) 1480.8 (s) 1389.8 (s) 1280.7 (s) 1200.9 (s) 1096.9 (s) 1054.27 (m). HRMS (EI) Calcd for $\mathrm{C}_{11} \mathrm{H}_{13} \mathrm{NO}$ : 175.0992. Found: 175.0997 .

$N$-(2-Vinyl-phenyl)-propionimidic acid ethyl ester: Analogous preparation as above,

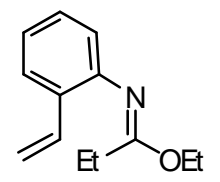
distilled at $65{ }^{\circ} \mathrm{C}$ at $100 \mathrm{mTorr}, 7.6 \mathrm{~g}, 38 \mathrm{mmol}, 76 \%$ yield. ${ }^{1} \mathrm{H}$ NMR (500 $\left.\mathrm{MHz}, \mathrm{C}_{6} \mathrm{D}_{6}\right): \delta 7.52(\mathrm{dd}, 1 \mathrm{H}, \mathrm{J}=1.2 \mathrm{~Hz}, \mathrm{~J}=7.6 \mathrm{~Hz}$, aryl CH) $7.05(\mathrm{~m}, 2 \mathrm{H}$, aryl $\left.\mathbf{C H} \& \mathbf{C H}=\mathrm{CH}_{2}\right) 6.93(\mathrm{td}, 1 \mathrm{H}, \mathrm{J}=7.3 \mathrm{~Hz}, \mathrm{~J}=0.6 \mathrm{~Hz}$, aryl $\mathbf{C H}) 6.69$ $(\mathrm{dd}, 1 \mathrm{H}, \mathrm{J}=1.2 \mathrm{~Hz}, 7.9 \mathrm{~Hz}$, aryl CH) $5.65(\mathrm{dd}, 1 \mathrm{H}, \mathrm{J}=1.5 \mathrm{~Hz}, 17.7 \mathrm{~Hz}$, $\left.\mathrm{CH}=\mathrm{CH}_{\mathrm{a}} \mathrm{H}_{\mathrm{b}}\right) 5.15\left(\mathrm{dd}, 1 \mathrm{H}, \mathrm{J}=1.5 \mathrm{~Hz}, 11.0 \mathrm{~Hz}, \mathrm{CH}=\mathrm{CH}_{\mathrm{a}} \mathbf{H}_{\mathrm{b}}\right) 4.21(\mathrm{q}, 2 \mathrm{H}, \mathrm{J}=7.1 \mathrm{~Hz}$, $\left.\mathrm{OCH}_{2} \mathrm{CH}_{3}\right) 1.98\left(\mathrm{q}, 2 \mathrm{H}, \mathrm{J}=7.6 \mathrm{~Hz}, \mathrm{CCH}_{2} \mathrm{CH}_{3}\right) 1.14\left(\mathrm{t}, 3 \mathrm{H}, \mathrm{J}=7.1 \mathrm{~Hz}, \mathrm{OCH}_{2} \mathrm{CH}_{3}\right) 0.90$ $\left(\mathrm{t}, 3 \mathrm{H}, \mathrm{J}=7.6 \mathrm{~Hz}, \mathrm{CCH}_{2} \mathrm{CH}_{3}\right) .{ }^{13} \mathrm{C} \mathrm{NMR}\left(125 \mathrm{MHz}, \mathrm{C}_{6} \mathrm{D}_{6}\right): \delta 164.6147 .8134 .4129 .7$ 129.1126 .4123 .6122 .0113 .961 .924 .214 .7 11.0. IR (NEAT, NaCl plates): 3085.8 (w) $2978.1(\mathrm{~s}) 2896.5(\mathrm{~m}) 1667.6$ (s) 1625.5 (m) 1596.9 (m) 1478.9 (m) 1364.8 (m) 1282.5 
(s) 1231.0 (s) 1179.2 (m) 1086.6 (s) 1058.9 (s) 1016.0 (s). HRMS (ESI ${ }^{+}$) Calcd for $\mathrm{C}_{13} \mathrm{H}_{17} \mathrm{NO}[\mathrm{M}+\mathrm{H}]: 204.1383$. Found: 204.1383.

$N$-(2-Vinyl-phenyl)-benzimidic acid ethyl ester: Analogous preparation as above, distilled at $90-100{ }^{\circ} \mathrm{C}$, at $100 \mathrm{mTorr}, 4.5 \mathrm{~g}, 19 \mathrm{mmol}, 56 \%$ yield. ${ }^{1} \mathrm{H}$ NMR $\left(500 \mathrm{MHz}, \mathrm{C}_{6} \mathrm{D}_{6}\right): \delta 7.50(\mathrm{dd}, 1 \mathrm{H}, \mathrm{J}=7.3 \mathrm{~Hz}, \mathrm{~J}=1.2 \mathrm{~Hz}$, aryl $\mathrm{CH})$ $7.39(\mathrm{dd}, 1 \mathrm{H}, \mathrm{J}=7.9 \mathrm{~Hz}, \mathrm{~J}=1.5 \mathrm{~Hz}$, aryl CH) $7.25(\mathrm{dd}, 1 \mathrm{H}, \mathrm{J}=17.7, \mathrm{~J}=$ $\left.11.0 \mathrm{~Hz}, \mathrm{CH}=\mathrm{CH}_{2}\right) 6.85(\mathrm{~m}, 5 \mathrm{H}$, aryl $\mathbf{C H}) 6.52(\mathrm{dd}, 1 \mathrm{H}, \mathrm{J}=1.5 \mathrm{~Hz}, 7.6 \mathrm{~Hz}$, aryl CH) $5.71\left(\mathrm{dd}, 1 \mathrm{H}, \mathrm{J}=1.5 \mathrm{~Hz}, 17.7 \mathrm{~Hz}, \mathrm{CH}=\mathrm{CH}_{\mathrm{a}} \mathrm{H}_{\mathrm{b}}\right) 5.19(\mathrm{dd}, 1 \mathrm{H}, \mathrm{J}=2.2 \mathrm{~Hz}, 11.0$ $\left.\mathrm{Hz}, \mathrm{CH}=\mathrm{CH}_{\mathrm{a}} \mathbf{H}_{\mathbf{b}}\right) 3.76\left(\mathrm{~s}, 3 \mathrm{H}, \mathrm{OCH}_{3}\right) .{ }^{13} \mathrm{C} \mathrm{NMR}\left(125 \mathrm{MHz}, \mathrm{C}_{6} \mathrm{D}_{6}\right): \delta 147.2134 .4132 .2$ 130.5130 .0129 .8129 .1126 .4123 .6122 .2114 .2 54.1 13.0. IR (NEAT, NaCl plates): 3084.7 (m) 3014.1 (m) 2943.1 (m) 2836.7 (w) 1664.9 (s) 1624.5 (s) 1595.2 (s) 1492.9 (m) 1413.2 (w) 1270.2 (s) 1191.9 (m) 1119.3 (s) 1074.4 (m) 1029.1 (m). HRMS (ESI $\left.{ }^{+}\right)$ Calcd for $\mathrm{C}_{16} \mathrm{H}_{15} \mathrm{NO}[\mathrm{M}+\mathrm{H}]:$ 238.1226. Found: 238.1228.

Pent-4-enoic acid (2-vinyl-phenyl)-amide: To a solution of 2-vinylaniline, (5 g, 42<smiles>C=CCCC(=O)Nc1ccccc1C=C</smiles>
mmol) pyridine $(4.3 \mathrm{~mL}, 46 \mathrm{mmol})$ and $\mathrm{N}, \mathrm{N}$-dimethylaminopyridine $(256 \mathrm{mg}, 2.1 \mathrm{mmol})$ in dichloromethane at $0{ }^{\circ} \mathrm{C}$ was added drop-wise 4-pentenoyl chloride $(5.1 \mathrm{~mL}, 46 \mathrm{mmol})$. After warming to room temperature, $10 \% \mathrm{HCl}$ in water $(100 \mathrm{~mL})$ was added to the solution. This mixture was extracted three times with dichloromethane $(200 \mathrm{~mL})$. All organic extracts were combined, washed with saturated $\mathrm{NaHCO}_{3}$ solution, then $\mathrm{NaCl}$ solution, dried over $\mathrm{MgSO}_{4}$ and concentrated in vacuo to an off-white solid. The solid was triterated in pentane for 1 hour, then filtered to afford a white powder, $6.94 \mathrm{~g}, 34 \mathrm{mmol}$, $82 \%$ yield. ${ }^{1} \mathrm{H}$ NMR $\left(500 \mathrm{MHz}, \mathrm{C}_{6} \mathrm{D}_{6}\right) \delta 7.95(\mathrm{~d}, 1 \mathrm{H}, \mathrm{J}=7.9 \mathrm{~Hz}$, aryl CH) $7.25(\mathrm{~d}, 1 \mathrm{H}, \mathrm{J}$ $=7.6 \mathrm{~Hz}$, aryl CH) $7.08(\mathrm{br}, 1 \mathrm{H}, \mathrm{NH}) 7.01(\mathrm{~m}, 1 \mathrm{H}$, aryl CH) $6.86(\mathrm{t}, 1 \mathrm{H}, \mathrm{J}=7.9 \mathrm{~Hz}$, aryl CH) $6.64\left(\mathrm{dd}, 1 \mathrm{H}, \mathrm{J}=11.0 \mathrm{~Hz}, 17.4 \mathrm{~Hz}, \mathrm{ArCH}=\mathrm{CH}_{2}\right) 5.67\left(\mathrm{~m}, 1 \mathrm{H}, \mathrm{CH}_{2} \mathrm{CH}=\mathrm{CH}_{2}\right) 5.42$ $\left(\mathrm{dd}, 1 \mathrm{H}, \mathrm{J}=1.5 \mathrm{~Hz}, 17.4 \mathrm{~Hz}, \operatorname{ArCH}=\mathrm{CH}_{\mathbf{a}} \mathrm{H}_{\mathrm{b}}\right) 5.07\left(\mathrm{~d}, 1 \mathrm{H}, \mathrm{J}=10.7 \mathrm{~Hz}, \mathrm{ArCH}=\mathrm{CH}_{\mathrm{a}} \mathbf{H}_{\mathbf{b}}\right)$ $4.94\left(\mathrm{~d}, 1 \mathrm{H}, \mathrm{J}=17.4 \mathrm{~Hz}, \mathrm{CH}_{2} \mathrm{CH}=\mathrm{CH}_{\mathbf{a}} \mathrm{H}_{\mathrm{b}}\right) 4.90\left(\mathrm{~d}, 1 \mathrm{H}, \mathrm{J}=10.7 \mathrm{~Hz}, \mathrm{CH}_{2} \mathrm{CH}=\mathrm{CH}_{\mathrm{a}} \mathbf{H}_{\mathbf{b}}\right)$ $2.27\left(\mathrm{~m}, 2 \mathrm{H}, \mathrm{C}(\mathrm{O}) \mathrm{CH}_{2} \mathrm{CH}_{2}\right) 1.94\left(\mathrm{t}, 2 \mathrm{H}, \mathrm{J}=7.5 \mathrm{~Hz}, \mathrm{C}(\mathrm{O}) \mathrm{CH}_{2} \mathrm{CH}_{2}\right) .{ }^{13} \mathrm{C} \mathrm{NMR}(125 \mathrm{MHz}$, $\left.\mathrm{C}_{6} \mathrm{D}_{6}\right) \delta 170.5137 .8135 .8133 .3131 .0129 .0128 .7127 .0125 .6124 .9117 .1115 .936 .5$ 30.1. IR (KBr): 3271.5 (s) 3078.2 (w) 2977.3 (w) 1827.2 (w) 1651.5 (s) 1528.8 (s) 1449.8 (s) $1373.2(\mathrm{~m}) 1291.3(\mathrm{~m}) 1197.3(\mathrm{~m}) 1022.1(\mathrm{w}) . \mathrm{HRMS}\left(\mathrm{ESI}^{-}\right)$Calcd for $\mathrm{C}_{13} \mathrm{H}_{15} \mathrm{NO}[\mathrm{M}$ $+\mathrm{Na}]: 224.1046$. Found: 224.1050 .

Pent-4-enoic acid (benzyl)-amide Prepared analogously to above: 9.35 g, 50 mmol, 90

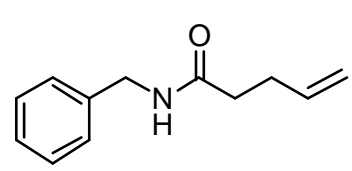
$\%$ yield. ${ }^{1} \mathrm{H}$ NMR $\left(500 \mathrm{MHz}, \mathrm{C}_{6} \mathrm{D}_{6}\right) \delta 7.09(\mathrm{~m}, 5 \mathrm{H}$, aryl CH) 5.73 $\left(\mathrm{m}, 1 \mathrm{H}, \mathrm{CH}_{2} \mathrm{CH}=\mathrm{CH}_{2}\right) 5.10(\mathrm{br}, 1 \mathrm{H}, \mathrm{NH}) 4.95\left(\mathrm{~m}, 2 \mathrm{H}, \mathrm{CH}=\mathrm{CH}_{2}\right)$ $4.21\left(\mathrm{~s}, 2 \mathrm{H}, \mathrm{CH}_{2} \mathrm{Ar}\right) 2.32\left(\mathrm{~m}, 2 \mathrm{H}, \mathrm{C}(\mathrm{O}) \mathrm{CH}_{2} \mathrm{CH}_{2}\right) 1.86(\mathrm{t}, 2 \mathrm{H}, \mathrm{J}=$ $\left.7.6 \mathrm{~Hz}, \mathrm{C}(\mathrm{O}) \mathrm{CH}_{2} \mathrm{CH}_{2}\right) .{ }^{13} \mathrm{C} \mathrm{NMR}\left(125 \mathrm{MHz}, \mathrm{C}_{6} \mathrm{D}_{6}\right) \delta 173.1139 .6$

137.9190 .0127 .7115 .743 .935 .7 30.5. IR (NEAT, NaCl plates): 3288.5 (s) 3031.7 (m) 1727.5 (m) 1640.8 (s) 1546.4 (s) 1453.9 (m) 1380.1 (m) 1266.2 (m) 1079.7 (m) 1027.9 (m). HRMS (ESI) Calcd for $\mathrm{C}_{12} \mathrm{H}_{15} \mathrm{NO}[\mathrm{M}+\mathrm{Na}]: 212.1046$. Found: 212.1047. 


\section{EXPERIMENTALS FOR SUbSTRATES AND PRODUCTS}

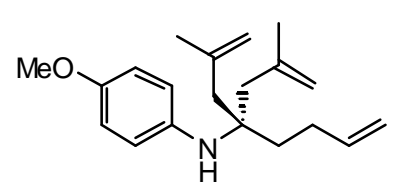

[1,1-Bis-(2-methyl-allyl)-pent-4-enyl]-(4-methoxy-phenyl)amine (1a) Phosphorous pentachloride $(2 \mathrm{~g}, 9.7 \mathrm{mmol})$ was added to a solution of pent-4-enoic acid (4-methoxy-phenyl)amide $(2 \mathrm{~g}, 9.7 \mathrm{mmol})$ and dichloromethane $(20 \mathrm{~mL})$ in a Schlenk flask under vigorous $\mathrm{N}_{2}$ flow, to produce a mild exotherm. After 20 minutes, the solvent was removed in vacuo to afford a green oil. ${ }^{1} \mathrm{H}$ NMR of the crude product indicated complete conversion to the imidoyl chloride. This material was diluted in THF $(20 \mathrm{~mL})$ and added to methallyl Grignard $(73 \mathrm{~mL}, 0.4 \mathrm{M})$ at room temperature to afford an orange solution. The mixture was quenched on water $(150 \mathrm{~mL})$ then extracted three times with ether $(200 \mathrm{~mL})$. All organic extracts were combined, washed with saturated solutions of $\mathrm{NH}_{4} \mathrm{Cl}$, then $\mathrm{NaCl}$, dried $\left(\mathrm{MgSO}_{4}\right)$ and concentrated in vacuo to a brown oil. The crude product was purified by column chromatography, eluting with $20 \%$ ether in pentane, to afford $2.23 \mathrm{~g}(7.5 \mathrm{mmol}, 77 \%)$ of yellow oil. ${ }^{1} \mathrm{H}$ NMR $\left(500 \mathrm{MHz}, \mathrm{C}_{6} \mathrm{D}_{6}\right) \delta$ $6.76(\mathrm{~d}, 2 \mathrm{H}, \mathrm{J}=9 \mathrm{~Hz}$, aryl $\mathbf{C H}) 6.55(\mathrm{~d}, 2 \mathrm{H}, \mathrm{J}=9 \mathrm{~Hz}$, aryl $\mathbf{C H}) 5.76\left(\mathrm{~m}, 1 \mathrm{H}, \mathrm{CH}=\mathrm{CH}_{2}\right)$ $5.05\left(\mathrm{dd}, 1 \mathrm{H}, \mathrm{J}=1.5 \mathrm{~Hz}, 17.1 \mathrm{~Hz}, \mathrm{CH}=\mathrm{CH}_{\mathrm{a}} \mathrm{H}_{\mathrm{b}}\right) 4.97(\mathrm{dd}, 1 \mathrm{H}, \mathrm{J}=1.2 \mathrm{~Hz}, 10.4 \mathrm{~Hz}$, $\left.\mathrm{CH}=\mathrm{CH}_{\mathrm{a}} \mathbf{H}_{\mathbf{b}}\right) 4.93\left(\mathrm{~m}, 2 \mathrm{H}, \mathrm{CH}_{3} \mathrm{C}=\mathrm{CH}_{\mathbf{a}} \mathrm{H}_{\mathrm{b}}\right) 4.85\left(\mathrm{~m}, 2 \mathrm{H}, \mathrm{CH}_{3} \mathrm{C}=\mathrm{CH}_{\mathrm{a}} \mathbf{H}_{\mathbf{b}}\right) 3.39(\mathrm{~s}, 3 \mathrm{H}$, $\left.\mathrm{CH}_{3} \mathrm{O}\right) 2.41\left(\mathrm{~d}, 2 \mathrm{H}, \mathrm{J}=13.9 \mathrm{~Hz}, \mathrm{C}\left(\mathrm{CH}_{\mathrm{a}} \mathrm{H}_{\mathrm{b}}\right)_{2}\right) 2.26\left(\mathrm{~d}, 2 \mathrm{H}, \mathrm{J}=13.9 \mathrm{~Hz}, \mathrm{C}\left(\mathrm{CH}_{\mathrm{a}} \mathbf{H}_{\mathbf{b}}\right)_{2}\right) 2.09$ $\left(\mathrm{m}, 2 \mathrm{H}, \mathrm{CH}_{2}\right) 1.74\left(\mathrm{~s}, 6 \mathrm{H}, \mathrm{CH}_{3} \mathrm{C}=\mathrm{CH}_{2}\right) 1.69\left(\mathrm{~m}, 2 \mathrm{H}, \mathrm{CH}_{2}\right) .{ }^{13} \mathrm{C} \mathrm{NMR}\left(125 \mathrm{MHz}, \mathrm{C}_{6} \mathrm{D}_{6}\right) \delta$

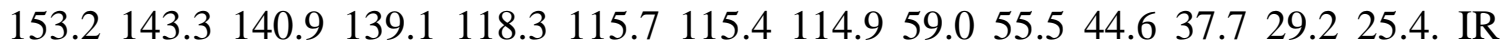
(NEAT, NaCl plates): 3406.1 (m) 3073.3 (m) 2945.4 (m) 1640.0 (m) 1510.3 (s) 1455.1 (m) 1373.5 (w) 1240.5 (s) 1179.4 (m) 1041.5 (m). HRMS (ESI) Calcd for $\mathrm{C}_{20} \mathrm{H}_{29} \mathrm{NO}$ [M $+\mathrm{H}]: 300.2322$. Found: 300.2318 .

[1,1-Bis-(2-methyl-allyl)-pent-4-enyl]-(4-bromo-phenyl)-amine (1b) Analogous

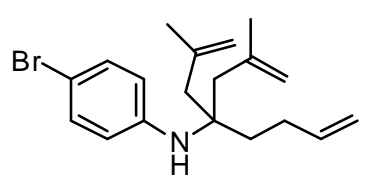
preparation as above: $2.2 \mathrm{~g}, 6.5 \mathrm{mmol}, 82 \%$ yield. ${ }^{1} \mathrm{H}$ NMR (500 $\left.\mathrm{MHz}, \mathrm{C}_{6} \mathrm{D}_{6}\right): \delta 7.15(\mathrm{~d}, 2 \mathrm{H}, \mathrm{J}=8.9 \mathrm{~Hz}$, aryl CH) $6.17(\mathrm{~d}, 2 \mathrm{H}, \mathrm{J}=$ $8.9 \mathrm{~Hz}$, aryl $\mathbf{C H}) 5.70\left(\mathrm{~m}, 1 \mathrm{H}, \mathrm{CH}=\mathrm{CH}_{2}\right) 5.00(\mathrm{dd}, 1 \mathrm{H}, \mathrm{J}=17.1$ $\left.\mathrm{Hz}, 0.6 \mathrm{~Hz}, \mathrm{CH}=\mathrm{CH}_{\mathrm{a}} \mathrm{H}_{\mathrm{b}}\right) 4.95(\mathrm{dd}, 1 \mathrm{H}, \mathrm{J}=10.1 \mathrm{~Hz}, 0.9 \mathrm{~Hz}$, $\left.\mathrm{CH}=\mathrm{CH}_{\mathrm{a}} \mathbf{H}_{\mathbf{b}}\right) 4.86\left(\mathrm{~s}, 2 \mathrm{H}, \mathrm{C}\left(\mathrm{CH}_{3}\right)=\mathrm{CH}_{\mathrm{a}} \mathrm{H}_{\mathrm{b}}\right) 4.72\left(\mathrm{~s}, 2 \mathrm{H}, \mathrm{C}\left(\mathrm{CH}_{3}\right)=\mathrm{CH}_{\mathrm{a}} \mathbf{H}_{\mathbf{b}}\right) 3.29(\mathrm{~s}, 3 \mathrm{H}$, $\left.\mathrm{CH}_{3} \mathrm{O}\right) 2.29\left(\mathrm{~d}, 2 \mathrm{H}, \mathrm{J}=14.3 \mathrm{~Hz}, \mathrm{CH}_{\mathbf{a}} \mathrm{H}_{\mathrm{b}}\right) 2.10\left(\mathrm{~d}, 2 \mathrm{H}, \mathrm{J}=14.4 \mathrm{~Hz}, \mathrm{CH}_{\mathrm{a}} \mathbf{H}_{\mathbf{b}}\right) 1.97(\mathrm{~m}, 2 \mathrm{H}$, $\left.\mathrm{CH}_{2}\right) 1.61\left(\mathrm{~s}, 6 \mathrm{H}, \mathrm{CH}_{3} \mathrm{C}\right) 1.57\left(\mathrm{~m}, 2 \mathrm{H}, \mathrm{CH}_{2}\right) .{ }^{13} \mathrm{C} \mathrm{NMR}\left(125 \mathrm{MHz}, \mathrm{C}_{6} \mathrm{D}_{6}\right): \delta 146.1142 .8$ 138.7132 .6127 .1117 .3115 .9115 .1109 .458 .844 .037 .2 29.0 25.1. IR (NEAT, NaCl plates): $3416.5(\mathrm{~m}) 3074.2$ (s) $2945.3(\mathrm{~s}) 1640.3$ (s) 1591.5 (s) 1488.7 (s) $1452.3(\mathrm{~m})$ $1373.5(\mathrm{~m}) 1318.9(\mathrm{~m}) 1254.3(\mathrm{~m}) 1180.8(\mathrm{w}) 1076.0$ (s). HRMS (ESI $\left.{ }^{+}\right)$Calcd for $\mathrm{C}_{19} \mathrm{H}_{26} \mathrm{NBr}[\mathrm{M}+\mathrm{H}]: 348.1321$. Found: 348.1308 .

[1,1-Bis-(2-methyl-allyl)-pent-4-enyl]-(2-methoxy-phenyl)-amine (1c) Analogous

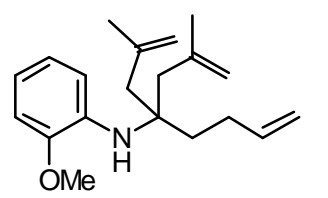
preparation as above: $0.72 \mathrm{~g}, 2.4 \mathrm{mmol}, 25 \%$ yield. ${ }^{1} \mathrm{H}$ NMR $(500$ $\left.\mathrm{MHz}, \mathrm{C}_{6} \mathrm{D}_{6}\right): \delta 6.92(\mathrm{~m}, 2 \mathrm{H}$, aryl CH) $6.70(\mathrm{~m}, 1 \mathrm{H}$, aryl CH) $6.56(\mathrm{~d}$, $1 \mathrm{H}, \mathrm{J}=7.6 \mathrm{~Hz}$, aryl $\mathbf{C H}) 5.76\left(\mathrm{~m}, 1 \mathrm{H}, \mathrm{CH}=\mathrm{CH}_{2}\right) 5.02(\mathrm{ddd}, 1 \mathrm{H}, \mathrm{J}=$ $\left.17.1 \mathrm{~Hz}, 1.5 \mathrm{~Hz}, \mathrm{~J}=3.66 \mathrm{~Hz}, \mathrm{CH}=\mathrm{CH}_{\mathbf{a}} \mathrm{H}_{\mathrm{b}}\right) 4.94(\mathrm{ddd}, 1 \mathrm{H}, \mathrm{J}=10.4$ $\left.\mathrm{Hz}, 1.2 \mathrm{~Hz}, \mathrm{~J}=2.1 \mathrm{~Hz}, \mathrm{CH}=\mathrm{CH}_{\mathrm{a}} \mathbf{H}_{\mathbf{b}}\right) 4.91\left(\mathrm{~m}, 2 \mathrm{H}, \mathrm{C}\left(\mathrm{CH}_{3}\right)=\mathrm{CH}_{\mathbf{a}} \mathrm{H}_{\mathrm{b}}\right)$ $4.84\left(\mathrm{~m}, 2 \mathrm{H}, \mathrm{C}\left(\mathrm{CH}_{3}\right)=\mathrm{CH}_{\mathrm{a}} \mathbf{H}_{\mathrm{b}}\right) 4.63(\mathrm{~s}, 1 \mathrm{H}, \mathrm{NH}) 3.30\left(\mathrm{~s}, 3 \mathrm{H}, \mathrm{CH}_{3} \mathrm{O}\right) 2.55(\mathrm{~d}, 2 \mathrm{H}, \mathrm{J}=14.4$ $\left.\mathrm{Hz}, \mathrm{CH}_{\mathbf{a}} \mathrm{H}_{\mathrm{b}}\right) 2.35\left(\mathrm{~d}, 2 \mathrm{H}, \mathrm{J}=14.4 \mathrm{~Hz}, \mathrm{CH}_{\mathrm{a}} \mathbf{H}_{\mathbf{b}}\right) 2.14\left(\mathrm{~m}, 2 \mathrm{H}, \mathrm{C} \mathbf{H}_{2}\right) 1.85\left(\mathrm{~m}, 2 \mathrm{H}, \mathrm{CH}_{2}\right) 1.75$ $\left(\mathrm{s}, 6 \mathrm{H}, \mathrm{CH}_{3} \mathrm{C}\right) .{ }^{13} \mathrm{C}$ NMR $\left(125 \mathrm{MHz}, \mathrm{C}_{6} \mathrm{D}_{6}\right)$ : $\delta 147.9143 .2139 .1137 .3127 .1121 .8116 .6$ 
Dolman, S. J. et. al, Supporting Information Page 6.

115.7 114.8 113.1 110.5 58.6 55.5 44.5 37.6 29.2 25.0. IR (NEAT, NaCl plates): 3427.7 (w) $3072.9(\mathrm{~m}) 29.46 .0(\mathrm{~s}) 2834.0(\mathrm{~m}) \mathbf{1 6 4 0 . 3}(\mathrm{s}) 1602.1(\mathrm{~s}) 1520.9(\mathrm{~s}) 1457.5(\mathrm{~s}) 1373.5$ (m) $1351.6(\mathrm{~m}) 1295.7$ (w) 1251.1 (s) 1221.4 (s) 1177.0 (m) 1124.5 (m) $1088.3(\mathrm{w})$ 1032.0 (s). HRMS (ESI ${ }^{+}$Calcd for $\mathrm{C}_{20} \mathrm{H}_{29} \mathrm{NO}[\mathrm{M}+\mathrm{Na}]$ : 322.2141 . Found: 322.2139 .

[1,1-Bis-(2-methyl-allyl)-pent-4-enyl]-(2-bromo-phenyl)-amine (1d) Analogous preparation as above: $2.4 \mathrm{~g}, 6.8 \mathrm{mmol}, 86 \%$ yield. ${ }^{1} \mathrm{H}$ NMR (500 $\left.\mathrm{MHz}, \mathrm{C}_{6} \mathrm{D}_{6}\right): \delta 7.38(\mathrm{dd}, 1 \mathrm{H}, \mathrm{J}=7.9 \mathrm{~Hz}, 1.5 \mathrm{~Hz}$, aryl CH) $6.94(\mathrm{dt}$, $1 \mathrm{H}, \mathrm{J}=7.8 \mathrm{~Hz}, 1.5 \mathrm{~Hz}$, aryl CH) $6.82(\mathrm{dd}, 1 \mathrm{H}, \mathrm{J}=8.2 \mathrm{~Hz}, \mathrm{~J}=1.2 \mathrm{~Hz}$, aryl $\mathrm{CH}) 6.35(\mathrm{dt}, 1 \mathrm{H}, \mathrm{J}=1.5 \mathrm{~Hz}, 7.6 \mathrm{~Hz}$, aryl CH) $5.71(\mathrm{~m}, 1 \mathrm{H}$, $\left.\mathrm{CH}=\mathrm{CH}_{2}\right) 5.00\left(\mathrm{dd}, 1 \mathrm{H}, \mathrm{J}=17.1 \mathrm{~Hz}, 1.5 \mathrm{~Hz}, \mathrm{CH}=\mathrm{CH}_{\mathbf{a}} \mathrm{H}_{\mathrm{b}}\right) 4.95(\mathrm{dd}, 1 \mathrm{H}, \mathrm{J}=10.1 \mathrm{~Hz}, 1.5$ $\left.\mathrm{Hz}, \mathrm{H}=\mathrm{CH}_{\mathrm{a}} \mathbf{H}_{\mathbf{b}}\right) 4.94\left(\mathrm{~s}, 2 \mathrm{H}, \mathrm{C}\left(\mathrm{CH}_{3}\right)=\mathrm{CH}_{\mathrm{a}} \mathrm{H}_{\mathrm{b}}\right) 4.89\left(\mathrm{~m}, 2 \mathrm{H}, \mathrm{C}\left(\mathrm{CH}_{3}\right)=\mathrm{CH}_{\mathrm{a}} \mathbf{H}_{\mathbf{b}}\right) 4.64(\mathrm{~s}, 1 \mathrm{H}$, NH) $2.42\left(\mathrm{~d}, 2 \mathrm{H}, \mathrm{J}=14.3 \mathrm{~Hz}, \mathrm{CH}_{\mathbf{a}} \mathrm{H}_{\mathrm{b}}\right) 2.29\left(\mathrm{~d}, 2 \mathrm{H}, \mathrm{J}=14.3 \mathrm{~Hz}, \mathrm{CH}_{\mathrm{a}} \mathbf{H}_{\mathbf{b}}\right) 2.06(\mathrm{~m}, 2 \mathrm{H}$, $\left.\mathrm{CH}_{2}\right) 1.80\left(\mathrm{~m}, 2 \mathrm{H}, \mathrm{CH}_{2}\right) 1.66\left(\mathrm{~s}, 6 \mathrm{H}, \mathrm{CH}_{3} \mathrm{C}\right) .{ }^{13} \mathrm{C} \mathrm{NMR}\left(125 \mathrm{MHz}, \mathrm{C}_{6} \mathrm{D}_{6}\right): \delta 144.1142 .6$ 138.7133 .7127 .1118 .0116 .4115 .0114 .2112 .059 .344 .6 37.5 29.1 25.0. IR (NEAT, $\mathrm{NaCl}$ plates): $3409.0(\mathrm{~m}) 3073.8(\mathrm{~m}) 2946.5(\mathrm{~m}) 1640.4(\mathrm{~m}) 01593.4(\mathrm{~s}) 1516.5(\mathrm{~s})$ 1463.1 (s) 1374.1 (w) 1318.8 (m) 1286.6 (m) 1261.1 (w) $1166.0(\mathrm{w}) 1087.7$ (m) 1017.6 (s). HRMS (ESI ${ }^{+}$) Calcd for $\mathrm{C}_{19} \mathrm{H}_{26} \mathrm{NBr}[\mathrm{M}+\mathrm{H}]$ : 348.1321. Found: 348.1331 .

[1,1-Bis-(2-methyl-allyl)-pent-4-enyl]-(3-triflouromethyl-phenyl)-amine

(1e)<smiles>C=CCC(CC=C)(CC(=C)C)Nc1cccc(C(F)(F)F)c1</smiles>
Analogous preparation as above: $2.5 \mathrm{~g}, 7.4 \mathrm{mmol}, 90 \%$ yield. ${ }^{1} \mathrm{H}$ NMR $\left(500 \mathrm{MHz}, \mathrm{C}_{6} \mathrm{D}_{6}\right): \delta 6.89(\mathrm{~m}, 3 \mathrm{H}$, aryl $\mathrm{CH}) 6.49(\mathrm{~m}, 1 \mathrm{H}$, aryl CH) $5.69\left(\mathrm{~m}, 1 \mathrm{H}, \mathrm{CH}=\mathrm{CH}_{2}\right) 5.02(\mathrm{ddd}, 1 \mathrm{H}, \mathrm{J}=17.1 \mathrm{~Hz}, 1.5 \mathrm{~Hz}, \mathrm{~J}=$ $\left.3.35 \mathrm{~Hz}, \mathrm{CH}=\mathrm{CH}_{\mathrm{a}} \mathrm{H}_{\mathrm{b}}\right) 4.96(\mathrm{ddd}, 1 \mathrm{H}, \mathrm{J}=10.1 \mathrm{~Hz}, 1.2 \mathrm{~Hz}, \mathrm{~J}=1.8 \mathrm{~Hz}$, $\left.\mathrm{CH}=\mathrm{CH}_{\mathrm{a}} \mathbf{H}_{\mathbf{b}}\right) 4.84\left(\mathrm{~m}, 2 \mathrm{H}, \mathrm{C}\left(\mathrm{CH}_{3}\right)=\mathrm{CH}_{\mathbf{a}} \mathrm{H}_{\mathrm{b}}\right) 4.71\left(\mathrm{~m}, 2 \mathrm{H}, \mathrm{C}\left(\mathrm{CH}_{3}\right)=\mathrm{CH}_{\mathrm{a}} \mathbf{H}_{\mathbf{b}}\right) 3.46(\mathrm{~s}, 3 \mathrm{H}$, $\left.\mathrm{CH}_{3} \mathrm{O}\right) 2.34\left(\mathrm{~d}, 2 \mathrm{H}, \mathrm{J}=14.3 \mathrm{~Hz}, \mathrm{CH}_{\mathbf{a}} \mathrm{H}_{\mathrm{b}}\right) 2.13\left(\mathrm{~d}, 2 \mathrm{H}, \mathrm{J}=14.3 \mathrm{~Hz}, \mathrm{CH}_{\mathrm{a}} \mathbf{H}_{\mathbf{b}}\right) 1.96(\mathrm{~m}, 2 \mathrm{H}$, $\left.\mathrm{CH}_{2}\right) 1.62\left(\mathrm{~m}, 2 \mathrm{H}, \mathrm{CH}_{2}\right) 1.59\left(\mathrm{~s}, 6 \mathrm{H}, \mathrm{CH}_{3} \mathrm{C}\right) .{ }^{13} \mathrm{C} \mathrm{NMR}\left(125 \mathrm{MHz}, \mathrm{C}_{6} \mathrm{D}_{6}\right): \delta 147.5142 .6$ $138.6130 .2118 .1116 .1115 .2113 .9(\mathrm{q}, \mathrm{J}=4 \mathrm{~Hz}) 112.158 .944 .037 .328 .9$ 25.1. IR (NEAT, NaCl plates): 3422.5 (w) 3076.2 (m) 2946.7 (s) 1641.2 (m) 1613.3 (s) 1526.4 (s) 1492.2 (s) 1439.1 (s) 1349.5 (s) 1278.8 (w) 1164.4 (s) 1097.2 (m) 1071.1 (s). HRMS $\left(\mathrm{ESI}^{+}\right.$) Calcd for $\mathrm{C}_{20} \mathrm{H}_{26} \mathrm{NF}_{3}[\mathrm{M}+\mathrm{H}]$ : 338.2090. Found: 338.2110.

[1,1-Bis-(2-methyl-allyl)-pent-4-enyl]-(benzyl)-amine (1f) Analogous preparation as

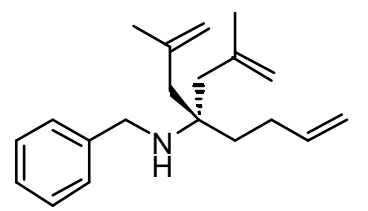
above: $1 \mathrm{~g}, 3.5 \mathrm{mmol}, 33 \%$ yield. ${ }^{1} \mathrm{H}$ NMR $\left(500 \mathrm{MHz}, \mathrm{C}_{6} \mathrm{D}_{6}\right) \delta$ $7.38(\mathrm{~d}, 2 \mathrm{H}, \mathrm{J}=7.5 \mathrm{~Hz}$, aryl $\mathbf{C H}) 7.22(\mathrm{t}, 2 \mathrm{H}, \mathrm{J}=7.5 \mathrm{~Hz}$, aryl $\mathbf{C H})$ $7.12(\mathrm{t}, 1 \mathrm{H}, \mathrm{J}=7.5 \mathrm{~Hz}$, aryl $\mathbf{C H}) 5.82\left(\mathrm{~m}, 1 \mathrm{H}, \mathrm{CH}=\mathrm{CH}_{2}\right) 5.08(\mathrm{dd}$, $\left.1 \mathrm{H}, \mathrm{J}=1.5 \mathrm{~Hz}, 17.1 \mathrm{~Hz}, \mathrm{CH}=\mathrm{CH}_{\mathrm{a}} \mathrm{H}_{\mathrm{b}}\right) 4.99(\mathrm{dd}, 1 \mathrm{H}, \mathrm{J}=1.2 \mathrm{~Hz}$, $\left.10.1 \mathrm{~Hz}, \mathrm{CH}=\mathrm{CH}_{\mathrm{a}} \mathbf{H}_{\mathbf{b}}\right) 4.92\left(\mathrm{~m}, 2 \mathrm{H}, \mathrm{CH}_{3} \mathrm{C}=\mathrm{CH}_{\mathrm{a}} \mathrm{H}_{\mathrm{b}}\right) 4.88(\mathrm{~m}, 2 \mathrm{H}$, $\left.\mathrm{CH}_{3} \mathrm{C}=\mathrm{CH}_{\mathrm{a}} \mathbf{H}_{\mathrm{b}}\right) 3.61\left(\mathrm{~s}, 2 \mathrm{H}, \mathrm{PhCH}_{2} \mathrm{~N}\right) 2.11(\mathrm{~s}, 1 \mathrm{H}, \mathrm{NH}) 2.10-2.06\left(\mathrm{~m}, 6 \mathrm{H}, \mathrm{CH}_{2}\right) 1.79(\mathrm{~s}$, 6H, $\left.\mathrm{CH}_{3}\right) 1.55\left(\mathrm{~m}, 2 \mathrm{H}, \mathrm{CH}_{2}\right) .{ }^{13} \mathrm{C}$ NMR (125 MHz, $\left.\mathrm{C}_{6} \mathrm{D}_{6}\right) \delta 143.4142 .0139 .6129 .0$ 128.8127 .5115 .2114 .658 .746 .743 .936 .7 29.1 25.8. IR (NEAT, NaCl plates): 3409.0 (br) $3073.6(\mathrm{~m}) 2940.9$ (s) 1639.8 (s) 1452.0 (s) 1373.2 (m) 1329.6 (m) 1241.9 (w) $1028.9(\mathrm{~m})$. HRMS (ESI ${ }^{+}$) Calcd for $\mathrm{C}_{20} \mathrm{H}_{29} \mathrm{~N}[\mathrm{M}+\mathrm{H}]:$ 284.2373. Found: 284.2374.

(2-Bromo-phenyl)-(1,1-diallyl-pent-4-enyl)-amine Analogous preparation as above: 2 g, $6.3 \mathrm{mmol}, 80 \%$ yield. ${ }^{1} \mathrm{H}$ NMR $\left(500 \mathrm{MHz}, \mathrm{C}_{6} \mathrm{D}_{6}\right): \delta 7.38(\mathrm{dd}, 1 \mathrm{H}, \mathrm{J}=7.9 \mathrm{~Hz}, \mathrm{~J}=1.5$ 


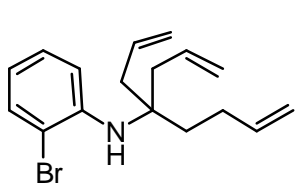

$\mathrm{Hz}$, aryl CH) $7.11(\mathrm{dt}, 1 \mathrm{H}, \mathrm{J}=7.3 \mathrm{~Hz}, \mathrm{~J}=1.2 \mathrm{~Hz}$, aryl CH) $6.92(\mathrm{dt}$, $1 \mathrm{H}, \mathrm{J}=1.2 \mathrm{~Hz}, \mathrm{~J}=8.2 \mathrm{~Hz}$, aryl $\mathrm{CH}) 6.79(\mathrm{dd}, 1 \mathrm{H}, \mathrm{J}=1.2 \mathrm{~Hz}, 7.6$ $\mathrm{Hz}$, aryl CH) $6.60(\mathrm{dd}, 1 \mathrm{H}, \mathrm{J}=1.2 \mathrm{~Hz}, 7.6 \mathrm{~Hz}$, aryl CH) $6.37(\mathrm{dt}$, $1 \mathrm{H}, \mathrm{J}=1.5 \mathrm{~Hz}, 7.5 \mathrm{~Hz}$, aryl $\mathrm{CH}) 5.69\left(\mathrm{~m}, 3 \mathrm{H}, \mathrm{CH}=\mathrm{CH}_{2}\right) 5.02-4.91$ $\left(\mathrm{m}, 6 \mathrm{H}, \mathrm{CH}=\mathrm{CH}_{2}\right) 4.44(\mathrm{br}, 1 \mathrm{H}, \mathrm{NH}) 2.31-2.18\left(\mathrm{~m}, 4 \mathrm{H}, \mathrm{CH}_{2}\right) 2.01-1.96(\mathrm{~m}, 2 \mathrm{H}, \mathrm{CH})$ $1.69-1.61\left(\mathrm{~m}, 2 \mathrm{H}, \mathrm{CH}_{2}\right) .{ }^{13} \mathrm{C}$ NMR (125 MHz, $\left.\mathrm{C}_{6} \mathrm{D}_{6}\right): \delta 139.0134 .5134 .0133 .6129 .8$ 128.8119 .2118 .7116 .9115 .0114 .6112 .658 .941 .636 .6 29.4. IR (NEAT, NaCl plates): 3394.7 (br m) 3075.7 (m) 2936.2 (m) 2861.1 (w) 1639.9 (m) 1593.4 (s) 1515.2 (s) 1463.1 (s) $1327.9(\mathrm{~m}) 1165.9(\mathrm{w}) 1018.6(\mathrm{~m})$.

(4-Methoxy-phenyl)-[3-methyl-1-(2-methyl-allyl)-cyclohex-3-enyl]-amine<smiles>C=C(C)C[C@]1(Nc2ccc(OC)cc2)CCC=C(C)C1</smiles>
Catalyst $3\left[(R) \operatorname{Pr}_{2} \mathrm{Bnz}_{2} \mathrm{Bitet}\right](5 \mathrm{mg}, 7 \mu \mathrm{mol})$ was added to a solution of $\mathbf{1 a}(68 \mathrm{mg}, 230 \mu \mathrm{mol})$ in benzene- $d_{6}(1 \mathrm{~mL})$, and the resultant mixture placed in a J. Young tube. The reaction was monitored by ${ }^{1} \mathrm{H}$ NMR analysis. After 1 hour, the reaction was complete. The mixture was exposed to air, stirred over charcoal, filtered through celite and concentrated in vacuo. The crude was purified by chromatography over silica gel, eluting with $1 \%$ ether in pentanes to afford $50 \mathrm{mg}$ of clear, colorless oil $\left(186 \mu \mathrm{mol}, 81 \%\right.$ yield.) ${ }^{1} \mathrm{H} \mathrm{NMR}$ $\left(500 \mathrm{MHz}, \mathrm{C}_{6} \mathrm{D}_{6}\right): \delta 6.75(\mathrm{~m}, 2 \mathrm{H}$, aryl $\mathrm{CH}) 6.60(\mathrm{~m}, 2 \mathrm{H}$, aryl $\mathrm{CH}) 5.35(\mathrm{~m}, 1 \mathrm{H}$, $\left.\mathrm{ArCH}=\mathrm{C}\left(\mathrm{CH}_{3}\right)\right) 4.96\left(\mathrm{~m}, 1 \mathrm{H}, \mathrm{CH}_{3} \mathrm{C}=\mathrm{CH}_{\mathbf{a}} \mathrm{H}_{\mathrm{b}}\right) 4.82\left(\mathrm{~m}, 1 \mathrm{H}, \mathrm{CH}_{3} \mathrm{C}=\mathrm{CH}_{\mathrm{a}} \mathbf{H}_{\mathbf{b}}\right) 3.39(\mathrm{~s}, 3 \mathrm{H}$, $\left.\mathrm{CH}_{3} \mathrm{O}\right) 3.08(\mathrm{br}, 1 \mathrm{H}, \mathrm{NH}) 2.54\left(\mathrm{~d}, 1 \mathrm{H}, \mathrm{J}=13.7 \mathrm{~Hz}, \mathrm{CCH}_{\mathrm{a}} \mathrm{H}_{\mathrm{b}} \mathrm{C}\left(\mathrm{CH}_{3}\right)=\right) 2.23(\mathrm{~d}, 1 \mathrm{H}, \mathrm{J}=$ $\left.13.7 \mathrm{~Hz}, \mathrm{CCH}_{\mathrm{a}} \mathbf{H}_{\mathrm{b}} \mathrm{C}\left(\mathrm{CH}_{3}\right)=\right) 1.97-1.85(\mathrm{~m}, 5 \mathrm{H}) 1.85\left(\mathrm{~s}, 3 \mathrm{H}, \mathrm{CH}_{3}\right) 1.56\left(\mathrm{~s}, 3 \mathrm{H}, \mathrm{CH}_{3}\right) 1.42$ (m, 1H). ${ }^{13} \mathrm{C}$ NMR (125 MHz, $\left.\mathrm{C}_{6} \mathrm{D}_{6}\right): \delta 153.8143 .6141 .1131 .5128 .7121 .3119 .5115 .2$ 115.1 55.5 46.4 42.9 31.7 25.4 24.2 23.3. IR (NEAT, NaCl plates): 3402.2 (w) 3069.7 (w) $2926.4(\mathrm{~s}) 1640.5(\mathrm{~m}) 1509.6$ (s) 1441.5 (m) 1375.2 (w) 1239.0 (s) 1179.3 (m) 1040.7 (m). HRMS (ESI) Calcd for $\mathrm{C}_{18} \mathrm{H}_{25} \mathrm{NO}[\mathrm{M}+\mathrm{H}]:$ 272.2009. Found: 272.2007. HPLC (Chiralpak AS, $0.1 \%$ IPA in Hexane, $254 \mathrm{~nm}, 1.0 \mathrm{~mL} / \mathrm{min}$ ) $67 \%$ e.e.

(4-Bromo-phenyl)-[3-methyl-1-(2-methyl-allyl)-cyclohex-3-enyl]-amine Analogous preparation as above, $57 \mathrm{mg}, 178 \mu \mathrm{mol}, 78 \%$ yield. ${ }^{1} \mathrm{H}$<smiles>CC(C)=C1C=CCC[C@@]1(Nc1ccc(Br)cc1)C1CCCCC1</smiles>
NMR (500 MHz, $\left.\mathrm{C}_{6} \mathrm{D}_{6}\right): \delta 7.17(\mathrm{~d}, 2 \mathrm{H}, \mathrm{J}=6.7 \mathrm{~Hz}$, aryl $\mathrm{CH}) 6.18(\mathrm{~d}$, $2 \mathrm{H}, \mathrm{J}=6.7 \mathrm{~Hz}$, aryl $\mathrm{CH}) 5.30\left(\mathrm{~m}, 1 \mathrm{H}, \mathrm{CH}=\mathrm{C}\left(\mathrm{CH}_{3}\right)\right) 4.91(\mathrm{~m}, 1 \mathrm{H}$, $\left.\mathrm{C}\left(\mathrm{CH}_{3}\right)=\mathrm{CH}_{\mathbf{a}} \mathrm{H}_{\mathrm{b}}\right) 4.70\left(\mathrm{~m}, 1 \mathrm{H}, \mathrm{C}\left(\mathrm{CH}_{3}\right)=\mathrm{CH}_{\mathrm{a}} \mathbf{H}_{\mathbf{b}}\right) 3.12(\mathrm{br}, 1 \mathrm{H}, \mathrm{NH})$ $2.45\left(\mathrm{~d}, 1 \mathrm{H}, \mathrm{J}=13.7 \mathrm{~Hz}, \mathrm{CH}_{\mathrm{a}} \mathrm{H}_{\mathrm{b}}\right) 2.08\left(\mathrm{~d}, 1 \mathrm{H}, \mathrm{J}=13.7 \mathrm{~Hz}, \mathrm{CH}_{\mathrm{a}} \mathbf{H}_{\mathbf{b}}\right) 1.92(\mathrm{br} \mathrm{d}, 1 \mathrm{H}, \mathrm{J}=$ $\left.15.9 \mathrm{~Hz}, \mathrm{CH}_{\mathbf{a}} \mathrm{H}_{\mathrm{b}}\right) 1.83-1.77\left(\mathrm{~m}, 3 \mathrm{H}, \mathbf{C H}_{2}, \mathrm{CH}_{\mathbf{a}} \mathrm{H}_{\mathrm{b}}\right) 1.82\left(\mathrm{br} \mathrm{d}, 1 \mathrm{H}, \mathrm{J}=17.8 \mathrm{~Hz}, \mathrm{CH}_{\mathrm{a}} \mathbf{H}_{\mathbf{b}}\right)$ $1.70\left(\mathrm{~s}, 3 \mathrm{H}, \mathrm{CH}_{3}\right) 1.53\left(\mathrm{~s}, 3 \mathrm{H}, \mathrm{CH}_{3}\right) 1.26\left(\mathrm{~m}, 1 \mathrm{H}, \mathrm{CH}_{\mathrm{a}} \mathbf{H}_{\mathbf{b}}\right) .{ }^{13} \mathrm{C} \mathrm{NMR}\left(125 \mathrm{MHz}, \mathrm{C}_{6} \mathrm{D}_{6}\right): \delta$ 146.5143 .0132 .5130 .8121 .7117 .4115 .3109 .655 .245 .343 .331 .125 .024 .1 23.0. IR (NEAT, NaCl): 3413.7 (m) 3071.8 (m) 2923.5 (s) 2849.6 (m) 1641.3 (m) 1591.1 (s) 1489.0 (s) 1444.4 (m) 1375.3 (m) 1317.1 (m) 1255.5 (m) 1180.0 (m) 1075.6 (m). HRMS (ESI ${ }^{-}$) Calcd for $\mathrm{C}_{7} \mathrm{H}_{22} \mathrm{NBr}[\mathrm{M}+\mathrm{Na}$ ]: 342.0828. Found: 342.0832. HPLC (Chiralpak AS, $0.1 \%$ IPA in Hexane, $254 \mathrm{~nm}, 1.0 \mathrm{~mL} / \mathrm{min}) 67 \%$ e.e.

(2-Methoxy-phenyl)-[3-methyl-1-(2-methyl-allyl)-cyclohex-3-enyl]-amine

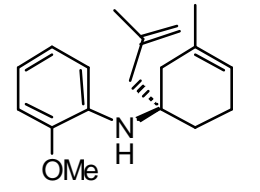
Analogous preparation as above, $62 \mathrm{mg}, 230 \mu \mathrm{mol}, 99 \%$ yield. ${ }^{1} \mathrm{H}$ NMR $\left(500 \mathrm{MHz}, \mathrm{C}_{6} \mathrm{D}_{6}\right): \delta 6.96(\mathrm{~m}, 2 \mathrm{H}$, aryl CH) $6.71(\mathrm{dt}, 1 \mathrm{H}, \mathrm{J}=1.8, \mathrm{~J}=7.9$ $\mathrm{Hz}$, aryl CH) $6.57(\mathrm{dd}, 1 \mathrm{H}, \mathrm{J}=1.2 \mathrm{~Hz}, 7.9 \mathrm{~Hz}$, aryl CH) $5.36(\mathrm{~m}, 1 \mathrm{H}$, 
$\left.\mathrm{CH}=\mathrm{C}\left(\mathrm{CH}_{3}\right)\right) 4.93\left(\mathrm{~m}, 1 \mathrm{H}, \mathrm{C}\left(\mathrm{CH}_{3}\right)=\mathrm{CH}_{\mathbf{a}} \mathrm{H}_{\mathrm{b}}\right) 4.77\left(\mathrm{~m}, 1 \mathrm{H}, \mathrm{C}\left(\mathrm{CH}_{3}\right)=\mathrm{CH}_{\mathrm{a}} \mathbf{H}_{\mathbf{b}}\right) 4.52(\mathrm{br}, 1 \mathrm{H}$, NH) $3.28\left(\mathrm{~s}, 3 \mathrm{H}, \mathrm{CH}_{3} \mathrm{O}\right) 2.70\left(\mathrm{~d}, 1 \mathrm{H}, \mathrm{J}=13.7 \mathrm{~Hz}, \mathrm{CH}_{\mathbf{a}} \mathrm{H}_{\mathrm{b}}\right) 2.33(\mathrm{~d}, 1 \mathrm{H}, \mathrm{J}=13.7 \mathrm{~Hz}$, $\left.\mathrm{CH}_{\mathrm{a}} \mathbf{H}_{\mathrm{b}}\right) 2.18-1.84\left(\mathrm{~m}, 5 \mathrm{H}, \mathrm{CH}_{2}, \mathrm{CH}_{\mathrm{a}} \mathrm{H}_{\mathrm{b}}\right) 1.81\left(\mathrm{~s}, 3 \mathrm{H}, \mathrm{CH}_{3}\right) 1.54\left(\mathrm{~s}, 3 \mathrm{H}, \mathrm{CH}_{3}\right) 1.49$ (m, $\left.1 \mathrm{H}, \mathrm{CH}_{\mathrm{a}} \mathbf{H}_{\mathbf{b}}\right) .{ }^{13} \mathrm{C}$ NMR (125 MHz, $\left.\mathrm{C}_{6} \mathrm{D}_{6}\right): \delta 148.0143 .5137 .6131 .3121 .8121 .5116 .6$ 115.1113 .1110 .555 .555 .045 .243 .531 .7 24.9 24.2 23.3. IR (NEAT, NaCl): $3427.1(\mathrm{w})$ $3069.8(\mathrm{~m}) 2923.4$ (s) $2834.6(\mathrm{~m}) 1641.0$ (m) 1601.6 (s) $1514.7(\mathrm{~s}) 1457.2(\mathrm{~s}) 1359.1(\mathrm{~m})$ 1252.0 (s) $1176.2(\mathrm{~m}) 1115.2(\mathrm{~m}) 1031.8$ (s). HRMS $\left(\mathrm{ESI}^{+}\right)$Calcd for $\mathrm{C}_{18} \mathrm{H}_{25} \mathrm{NO}[\mathrm{M}+$ H]: 272.2009. Found: 272.2012. HPLC (Chiralcel OD, 0.1\% IPA in Hexane, 254 nm, 1.0 $\mathrm{mL} / \mathrm{min}) 79 \%$ e.e.

(2-Bromo-phenyl)-[3-methyl-1-(2-methyl-allyl)-cyclohex-3-enyl]-amine

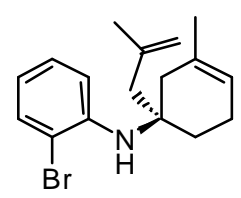
Analogous preparation as above, $160 \mathrm{mg}, 0.45 \mathrm{mmol}, 91 \%$ yield. ${ }^{1} \mathrm{H}$ NMR (500 MHz, $\left.\mathrm{C}_{6} \mathrm{D}_{6}\right): \delta 7.39(\mathrm{dt}, 1 \mathrm{H}, \mathrm{J}=1.8 \mathrm{~Hz}, 7.9 \mathrm{~Hz}$, aryl CH) 6.96 $(\mathrm{dt}, 1 \mathrm{H}, \mathrm{J}=1.5, \mathrm{~J}=8.2 \mathrm{~Hz}$, aryl $\mathrm{CH}) 6.85(\mathrm{dd}, 1 \mathrm{H}, \mathrm{J}=1.5 \mathrm{~Hz}, 8.2 \mathrm{~Hz}$, aryl CH) $6.36(\mathrm{dt}, 1 \mathrm{H}, \mathrm{J}=1.5 \mathrm{~Hz}, 7.9 \mathrm{~Hz}$, aryl $\mathbf{C H}) 5.33(\mathrm{~m}, 1 \mathrm{H}$, $\left.\mathrm{CH}=\mathrm{C}\left(\mathrm{CH}_{3}\right)\right) \quad 4.90 \quad\left(\mathrm{~m}, \quad 1 \mathrm{H}, \quad \mathrm{C}\left(\mathrm{CH}_{3}\right)=\mathrm{CH}_{\mathrm{a}} \mathrm{H}_{\mathrm{b}}\right) \quad 4.73 \quad(\mathrm{~m}, \quad 1 \mathrm{H}$, $\left.\mathrm{C}\left(\mathrm{CH}_{3}\right)=\mathrm{CH}_{\mathrm{a}} \mathbf{H}_{\mathbf{b}}\right) 4.50(\mathrm{br}, 1 \mathrm{H}, \mathrm{NH}) 2.55\left(\mathrm{~d}, 1 \mathrm{H}, \mathrm{J}=13.7 \mathrm{~Hz}, \mathrm{CH}_{\mathbf{a}} \mathrm{H}_{\mathrm{b}}\right) 2.23(\mathrm{~d}, 1 \mathrm{H}, \mathrm{J}=$ $\left.13.7 \mathrm{~Hz}, \mathrm{CH}_{\mathrm{a}} \mathbf{H}_{\mathbf{b}}\right) 2.09$ (br d, $\left.1 \mathrm{H}, \mathrm{J}=17.4 \mathrm{~Hz}, \mathrm{CH}_{\mathbf{a}} \mathrm{H}_{\mathrm{b}}\right) 2.02-1.78\left(\mathrm{~m}, 5 \mathrm{H}, \mathrm{CH}_{2}, \mathrm{CH}_{\mathbf{a}} \mathrm{H}_{\mathrm{b}}\right)$ $1.73\left(\mathrm{~s}, 3 \mathrm{H}, \mathrm{CH}_{3}\right) 1.54\left(\mathrm{~s}, 3 \mathrm{H}, \mathrm{CH}_{\mathbf{3}}\right) 1.38\left(\mathrm{~m}, 1 \mathrm{H}, \mathrm{CH}_{\mathrm{a}} \mathbf{H}_{\mathrm{b}}\right) \cdot{ }^{13} \mathrm{C} \mathrm{NMR}\left(125 \mathrm{MHz}, \mathrm{C}_{6} \mathrm{D}_{6}\right): \delta$ 144.5142 .8133 .6130 .9121 .6118 .0115 .6114 .2112 .255 .745 .243 .031 .224 .824 .1 23.2. IR (NEAT, $\mathrm{NaCl}$ ): 3406.8 (m) 3071.1 (w) 2963.9 (m) 2923.6 (m) 2849.6 (m) 1641.6 (m) 1593.4 (s) 1515.2 (s) 1463.2 (s) $1375.4(\mathrm{w}) 1323.4$ (m) 1286.6 (m) 1099.3 (m) 1018.1 (s). HRMS (ESI ${ }^{-}$) Calcd for $\mathrm{C}_{17} \mathrm{H}_{22} \mathrm{NBr}[\mathrm{M}+\mathrm{Na}]$ : 342.0828. Found: 342.0838. HPLC (Chiralcel OD, $0.1 \%$ IPA in Hexane, 254 nm, 1.0 mL/min) 81\% e.e.

(3-Triflouromethyl-phenyl)-[3-methyl-1 -(2-methyl-allyl)-cyclohex-3-enyl]-amine (2e)<smiles>CC(C)=CC[C@]1(Nc2cccc(C(F)(F)F)c2)CCC=C(C)C1</smiles>
Analogous preparation as above, $60 \mathrm{mg}, 191 \mu \mathrm{mol}, 85 \%$ yield. ${ }^{1} \mathrm{H}$ NMR $\left(500 \mathrm{MHz}, \mathrm{C}_{6} \mathrm{D}_{6}\right): \delta 6.90(\mathrm{~m}, 2 \mathrm{H}$, aryl $\mathrm{CH}) 6.83(\mathrm{~s}, 1 \mathrm{H}$, aryl $\mathrm{CH}) 6.48(\mathrm{~m}$, $1 \mathrm{H}$, aryl $\mathbf{C H}) 5.27\left(\mathrm{~m}, 1 \mathrm{H}, \mathbf{C H}=\mathrm{C}\left(\mathrm{CH}_{3}\right)\right) 4.88\left(\mathrm{~m}, 1 \mathrm{H}, \mathrm{C}\left(\mathrm{CH}_{3}\right)=\mathrm{CH}_{\mathbf{a}} \mathrm{H}_{\mathrm{b}}\right)$ $4.68\left(\mathrm{~m}, 1 \mathrm{H}, \mathrm{C}\left(\mathrm{CH}_{3}\right)=\mathrm{CH}_{\mathrm{a}} \mathbf{H}_{\mathbf{b}}\right) 3.31(\mathrm{br}, 1 \mathrm{H}, \mathbf{N H}) 2.49(\mathrm{~d}, 1 \mathrm{H}, \mathrm{J}=13.7$ $\left.\mathrm{Hz}, \mathrm{CH}_{\mathrm{a}} \mathrm{H}_{\mathrm{b}}\right) 2.11\left(\mathrm{~d}, 1 \mathrm{H}, \mathrm{J}=13.7 \mathrm{~Hz}, \mathrm{CH}_{\mathrm{a}} \mathbf{H}_{\mathbf{b}}\right) 1.93\left(\mathrm{~m}, 1 \mathrm{H}, \mathrm{CH}_{\mathbf{a}} \mathrm{H}_{\mathrm{b}}\right) 1.85-1.7(\mathrm{~m}, 4 \mathrm{H}$, $\left.\mathrm{CH}_{2}\right) 1.66\left(\mathrm{~s}, 3 \mathrm{H}, \mathrm{CH}_{3}\right) 1.52\left(\mathrm{~s}, 3 \mathrm{H}, \mathbf{C H}_{3}\right) 1.29\left(\mathrm{~m}, 1 \mathrm{H}, \mathrm{CH}_{\mathrm{a}} \mathbf{H}_{\mathbf{b}}\right) .{ }^{13} \mathrm{C}$ NMR $(125 \mathrm{MHz}$, $\left.\mathrm{C}_{6} \mathrm{D}_{6}\right): \delta 147.9142 .8130 .7130 .1121 .7118 .0115 .5113 .9112 .055 .345 .243 .331 .125 .0$ 24.0 23.0. IR (NEAT, NaCl plates): 3423.1 (m) 3073.9 (m) 2924.9 (s) 1642.6 (m) 1613.0 (s) 1524.2 (s) 1491.9 (s) 1439.9 (s) 1339.1 (s) 1263.8 (s) 1163.8 (s) 1124.4 (s) 1070.8 (s). HRMS $\left(\mathrm{ESI}^{+}\right)$Calcd for $\mathrm{C}_{18} \mathrm{H}_{22} \mathrm{NF}_{3}[\mathrm{M}+\mathrm{H}]$ : 310.1777. Found: 310.1769. HPLC (Chiralcel OD, $0.1 \%$ IPA in Hexane, $254 \mathrm{~nm}, 1.0 \mathrm{~mL} / \mathrm{min}$ ) $64 \%$ e.e.

\section{Benzyl-[3-methyl-1-(2-methyl-allyl)-cyclohex-3-enyl]-amine (2f) Analogous}

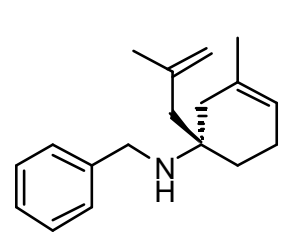

preparation as above, catalyst 4 used, $25 \mathrm{mg}, 96 \mu \mathrm{mol}, 85 \%$ yield. ${ }^{1} \mathrm{H}$ NMR $\left(500 \mathrm{MHz}, \mathrm{C}_{6} \mathrm{D}_{6}\right): \delta 7.39(\mathrm{~d}, 2 \mathrm{H}, \mathrm{J}=7.3 \mathrm{~Hz}$, aryl CH) $7.20(\mathrm{t}$, $2 \mathrm{H}, \mathrm{J}=7.3 \mathrm{~Hz}$, aryl CH) $7.13(\mathrm{t}, 2 \mathrm{H}, \mathrm{J}=7.3 \mathrm{~Hz}$, aryl CH) $5.33(\mathrm{~m}, 1 \mathrm{H}$, $\left.\operatorname{ArCH}=\mathrm{CCH}_{3} \mathrm{R}\right) 4.95\left(\mathrm{~m}, 1 \mathrm{H}, \mathrm{CH}_{3} \mathrm{C}=\mathrm{CH}_{\mathrm{a}} \mathrm{H}_{\mathrm{b}}\right) 4.81(\mathrm{~m}, 1 \mathrm{H}$, $\left.\mathrm{CH}_{3} \mathrm{C}=\mathrm{CH}_{\mathrm{a}} \mathbf{H}_{\mathbf{b}}\right) 3.63\left(\mathrm{~m}, 2 \mathrm{H}, \mathrm{NCH}_{2} \mathrm{Ph}\right) 2.25(\mathrm{~d}, 1 \mathrm{H}, \mathrm{J}=13.7 \mathrm{~Hz}$, $\left.\mathrm{CCH}_{\mathrm{a}} \mathrm{H}_{\mathrm{b}} \mathrm{C}\left(\mathrm{CH}_{3}\right)=\right) 2.05\left(\mathrm{~d}, 1 \mathrm{H}, \mathrm{J}=13.7 \mathrm{~Hz}, \mathrm{CCH}_{\mathrm{a}} \mathbf{H}_{\mathrm{b}} \mathrm{C}\left(\mathrm{CH}_{3}\right)=\right) 2.02-1.87(\mathrm{~m}, 4 \mathrm{H}) 1.88$ $\left(\mathrm{s}, 3 \mathrm{H}, \mathrm{CH}_{3}\right) 1.61(\mathrm{~m}, 1 \mathrm{H}) 1.57\left(\mathrm{~s}, 3 \mathrm{H}, \mathrm{CH}_{3}\right) 1.37(\mathrm{~m}, 1 \mathrm{H}) 1.11(\mathrm{t}, 1 \mathrm{H}, \mathrm{J}=7.0 \mathrm{~Hz},) .{ }^{13} \mathrm{C}$ 
NMR (125 MHz, $\left.\mathrm{C}_{6} \mathrm{D}_{6}\right): \delta 143.8142 .4132 .2128 .9127 .4120 .7114 .854 .546 .744 .6$ 42.0 31.6 25.6 24.3 23.4. IR (NEAT, NaCl plates): 3404.7 (w) 3027.9 (m) 2923.8 (s) 1640.0 (m) 1451.4 (m) 1375.2 (m) 1114.3 (m) 1070.9 (w) 1028.7 (m). HRMS (ESI) Calcd for $\mathrm{C}_{18} \mathrm{H}_{25} \mathrm{~N}[\mathrm{M}+\mathrm{H}]: 256.2060$. Found: 256.2056.

(1-Allyl-cyclohex-3-enyl)-(2-bromo-phenyl)-amine Analogous preparation as above, 50<smiles>Brc1ccccc1NC1CC2CCC1CC2</smiles>
mg, $170 \mu \mathrm{mol}, 95 \% .{ }^{1} \mathrm{H}$ NMR $\left(500 \mathrm{MHz}, \mathrm{C}_{6} \mathrm{D}_{6}\right): \delta 7.39(\mathrm{dd}, 1 \mathrm{H}, \mathrm{J}=1.2$ $\mathrm{Hz}, 7.9 \mathrm{~Hz}$, aryl CH) $6.94(\mathrm{dt}, 1 \mathrm{H}, \mathrm{J}=1.5, \mathrm{~J}=7.8 \mathrm{~Hz}$, aryl CH) $6.79(\mathrm{dd}$, $1 \mathrm{H}, \mathrm{J}=1.5 \mathrm{~Hz}, 8.2 \mathrm{~Hz}$, aryl CH) $6.38(\mathrm{dt}, 1 \mathrm{H}, \mathrm{J}=1.5 \mathrm{~Hz}, 7.6 \mathrm{~Hz}$, aryl CH) $5.76(\mathrm{~m}, 1 \mathrm{H}, \mathbf{C H}=\mathrm{CH}) 5.59\left(\mathrm{~m}, 1 \mathrm{H}, \mathrm{CH}_{\mathrm{a}}=\mathrm{CH}_{\mathrm{b}}\right) 5.43(\mathrm{~m}, 1 \mathrm{H}$, $\left.\mathrm{CH}_{\mathrm{a}}=\mathrm{CH}_{\mathrm{b}}\right) 4.98\left(\mathrm{~m}, 2 \mathrm{H}, \mathrm{CH}=\mathrm{CH}_{\mathbf{a}} \mathrm{H}_{\mathrm{b}}\right) 4.49(\mathrm{br}, 1 \mathrm{H}, \mathrm{NH}) 2.43(\mathrm{dd}, 1 \mathrm{H}, \mathrm{J}=14.5 \mathrm{~Hz}, \mathrm{~J}=$ $\left.7.3 \mathrm{~Hz}, \mathrm{CH}_{\mathrm{a}} \mathrm{H}_{\mathrm{b}}\right) 2.24\left(\mathrm{dd}, 1 \mathrm{H}, \mathrm{J}=14.5 \mathrm{~Hz}, \mathrm{~J}=7.3 \mathrm{~Hz}, \mathrm{CH}_{\mathrm{a}} \mathbf{H}_{\mathbf{b}}\right) 2.15(\mathrm{br} \mathrm{d}, 1 \mathrm{H}, \mathrm{J}=17.7$ $\left.\mathrm{Hz}, \mathrm{CH}_{\mathbf{a}} \mathrm{H}_{\mathrm{b}}\right) 2.00-1.70\left(\mathrm{~m}, 4 \mathrm{H}, \mathbf{C H}_{2}, \mathrm{CH}_{\mathbf{a}} \mathrm{H}_{\mathrm{b}}\right) 1.38\left(\mathrm{~m}, 1 \mathrm{H}, \mathrm{CH}_{\mathbf{a}} \mathrm{H}_{\mathrm{b}}\right) .{ }^{13} \mathrm{C}$ NMR $(125$ $\left.\mathrm{MHz}, \mathrm{C}_{6} \mathrm{D}_{6}\right): \delta 134.3133 .6129 .7127 .5118 .4116 .9114 .555 .042 .237 .9$ 31.1 23.2. IR (NEAT, NaCl): 3403.8 (m) 3072.9 (m) 2921.1 (m) 1638.9 (m) 1593.5 (s) 1513.4 (s) 1483.2 (s) 1326.2 (m) 1286.4 (m) 1131.0 (w) 1018.1 (m). HPLC (Chiralcel OD, $0.1 \%$ IPA in Hexane, $254 \mathrm{~nm}, 1.0 \mathrm{~mL} / \mathrm{min})<5 \%$ e.e.

[3-Methyl-1-(2-methyl-allyl)-but-3-enyl]-(2-vinyl-phenyl)-amine (6a) Styrenyl imidate ester $(1.62 \mathrm{~g}, 9.2 \mathrm{mmol})$ was added via syringe to methallyl Grignard (58<smiles>C=Cc1ccccc1NCC(=C)CC(=C)C</smiles>
$\mathrm{mL}, 0.34 \mathrm{M})$ at $-78{ }^{\circ} \mathrm{C}$. After 1 minute, TLC of an aliquot indicated the reaction to be complete, and it was quenched by addition of $1 \mathrm{~mL}$ of methanol. After the mixture had warmed to room temperature, $10 \% \mathrm{HCl}$ in water was added. The biphasic mixture was extracted three times with ether. All organic extracts were combined, washed with saturated $\mathrm{NaHCO}_{3}$ solution (50 $\mathrm{mL}$ ), then $\mathrm{NaCl}$ solution $(50 \mathrm{~mL})$, dried over $\mathrm{MgSO}_{4}$ and concentrated in vacuo to a yellow oil. Purification by column chromatography over silica, eluting with $1 \%$ ether in pentane afford $1.8 \mathrm{~g}$ of clear, bright yellow oil, $7.5 \mathrm{mmol}, 82 \%$ yield. ${ }^{1} \mathrm{H}$ NMR (500 $\left.\mathrm{MHz}, \mathrm{C}_{6} \mathrm{D}_{6}\right) \delta 7.32(\mathrm{dd}, 1 \mathrm{H}, \mathrm{J}=1.5 \mathrm{~Hz}, 7.3 \mathrm{~Hz}$, aryl CH) $7.19(\mathrm{~m}, 1 \mathrm{H}$, aryl CH) $6.79(\mathrm{dd}$, $\left.1 \mathrm{H}, \mathrm{J}=11.0 \mathrm{~Hz}, 17.4 \mathrm{~Hz}, \mathrm{CH}=\mathrm{CH}_{2}\right) 6.73(\mathrm{~m}, 1 \mathrm{H}, \operatorname{aryl~CH}) 5.56(\mathrm{dd}, 1 \mathrm{H}, \mathrm{J}=1.5 \mathrm{~Hz}, 17.4$ $\left.\mathrm{Hz}, \mathrm{CH}=\mathrm{CH}_{\mathbf{a}} \mathrm{H}_{\mathrm{b}}\right) 5.17\left(\mathrm{dd}, 1 \mathrm{H}, \mathrm{J}=1.5 \mathrm{~Hz}, 11.0 \mathrm{~Hz}, \mathrm{CH}=\mathrm{CH}_{\mathrm{a}} \mathbf{H}_{\mathbf{b}}\right) 4.79(\mathrm{~m}, 2 \mathrm{H}$, $\left.\mathrm{CH}_{3} \mathrm{C}=\mathrm{CH}_{\mathbf{a}} \mathrm{H}_{\mathrm{b}}\right) 4.75\left(\mathrm{~m}, 2 \mathrm{H}, \mathrm{CH}_{3} \mathrm{C}=\mathrm{CH}_{\mathrm{a}} \mathbf{H}_{\mathbf{b}}\right) 3.76(\mathrm{br}, 1 \mathrm{H}, \mathrm{NH}) 3.65$ (pentet, $1 \mathrm{H}, \mathrm{J}=6.7$ $\left.\mathrm{Hz}, \mathrm{CH}\left(\mathrm{CH}_{2} \mathrm{R}\right)_{2}\right) 2.15\left(\mathrm{~m}, 4 \mathrm{H}, \mathrm{CH}\left(\mathrm{CH}_{2} \mathrm{R}\right)_{2}\right) 1.56\left(\mathrm{~s}, 6 \mathrm{H}, \mathrm{CH}_{3} \mathrm{C}=\mathrm{CH}_{2}\right) \cdot{ }^{13} \mathrm{C}$ NMR $(125$ $\left.\mathrm{MHz}, \mathrm{C}_{6} \mathrm{D}_{6}\right) \delta 145.4143 .6134 .0129 .8128 .7125 .3117 .9116 .3113 .6111 .349 .643 .7$ 22.7. IR (NEAT, NaCl plates) 3419.9 (m) 3074.2 (m) 2933.3 (m) 1647.4 (m) $1602.2(\mathrm{~s})$ 1508.9 (s) 1458.5 (s) 1374.7 (m) 1316.5 (s) 1260.1 (m) 1185.6 (m) HRMS (EI $\left.{ }^{+}\right)$Calcd for $\mathrm{C}_{17} \mathrm{H}_{23} \mathrm{~N}$ : 241.1825. Found: 241.1822 .

[1-Ethyl-3-methyl-1-(2-methyl-allyl)-but-3-enyl]-(2-vinyl-phenyl)-amine

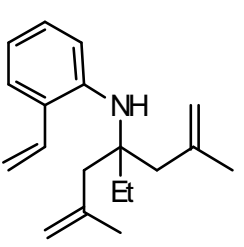
Analogous preparation as above, $400 \mathrm{mg}, 1.6 \mathrm{mmol}, 15 \%$ yield. ${ }^{1} \mathrm{H}$ NMR (500 MHz, $\left.\mathrm{C}_{6} \mathrm{D}_{6}\right): \delta 7.31(\mathrm{dd}, 1 \mathrm{H}, \mathrm{J}=7.6 \mathrm{~Hz}, \mathrm{~J}=1.5 \mathrm{~Hz}$, aryl $\mathrm{CH}$ ) $7.13(\mathrm{t}, 1 \mathrm{H}, \mathrm{J}=8.2 \mathrm{~Hz}$, aryl CH) $6.88(\mathrm{~d}, 1 \mathrm{H}, \mathrm{J}=8.2 \mathrm{~Hz}$, aryl $\mathrm{CH}) 6.73$ $\left(\mathrm{m}, 2 \mathrm{H}\right.$, aryl $\left.\mathbf{C H} \& \mathbf{C H}=\mathrm{CH}_{2}\right) 5.50(\mathrm{dd}, 1 \mathrm{H}, \mathrm{J}=1.8 \mathrm{~Hz}, \mathrm{~J}=17.4 \mathrm{~Hz}$, $\left.\mathrm{CH}=\mathrm{CH}_{\mathrm{a}} \mathrm{H}_{\mathrm{b}}\right) 5.12\left(\mathrm{dd}, 1 \mathrm{H}, \mathrm{J}=1.5 \mathrm{~Hz}, \mathrm{~J}=11.0 \mathrm{~Hz}, \mathrm{CH}=\mathrm{CH}_{\mathrm{a}} \mathbf{H}_{\mathbf{b}}\right) 4.89(\mathrm{~m}$, $\left.2 \mathrm{H}, \mathrm{C}=\mathrm{CH}_{\mathbf{a}} \mathrm{H}_{\mathrm{b}}\right) 4.79\left(\mathrm{~m}, 2 \mathrm{H}, \mathrm{C}=\mathrm{CH}_{\mathrm{a}} \mathbf{H}_{\mathbf{b}}\right) 3.90(\mathrm{br} \mathrm{s}, 1 \mathrm{H}, \mathrm{NH}) 2.50(\mathrm{~d}, 2 \mathrm{H}$, $\left.\mathrm{J}=14.4 \mathrm{~Hz}, \mathrm{CH}_{\mathrm{a}} \mathrm{H}_{\mathrm{b}}\right) 2.28\left(\mathrm{~d}, 2 \mathrm{H}, \mathrm{J}=14.4 \mathrm{~Hz}, \mathrm{CH}_{\mathrm{a}} \mathbf{H}_{\mathrm{b}}\right) 1.68\left(\mathrm{~s}, 6 \mathrm{H}, \mathrm{CH}_{3}\right) 1.64(\mathrm{q}, 2 \mathrm{H}, \mathrm{J}=$ 
$\left.7.5 \mathrm{~Hz}, \mathrm{CH}_{2} \mathrm{CH}_{3}\right) 0.80\left(\mathrm{t}, 3 \mathrm{H}, \mathrm{J}=7.5 \mathrm{~Hz}, \mathrm{CH}_{2} \mathrm{CH}_{3}\right) .{ }^{13} \mathrm{C} \mathrm{NMR}\left(125 \mathrm{MHz}, \mathrm{C}_{6} \mathrm{D}_{6}\right): \delta 143.2$ 134.5129 .3129 .0117 .5116 .9115 .9114 .243 .930 .8 25.1 9.2. IR (NEAT, NaCl plates): 3415.5 (m) 3072.7 (m) 2967.8 (s) 1639.6 (m) 1602.5 (s) 1514,3 (s) 1460.8 (s) 1373.67 (m) 1317.1 (s) $1282.7(\mathrm{~m}) 1162.0(\mathrm{~m}) \mathbf{1 0 2 7 . 0}(\mathrm{w})$. HRMS (ESI ${ }^{-}$) Calcd for $\mathrm{C}_{19} \mathrm{H}_{27} \mathrm{~N}[\mathrm{M}+$ $\mathrm{Na}$ : 292.2036. Found: 292.2032.

[3-Methyl-1-(2-methyl-allyl)-1-phenyl-but-3-enyl]-(2-vinyl-phenyl)-amine

(6c)

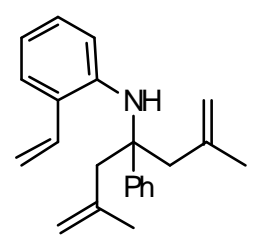

Prepared as above, $150 \mathrm{mg}, 0.46 \mathrm{mmol}, 11 \%$ yield. ${ }^{1} \mathrm{H} \mathrm{NMR}(500 \mathrm{MHz}$, $\left.\mathrm{C}_{6} \mathrm{D}_{6}\right): \delta 7.33(\mathrm{dd}, 1 \mathrm{H}, \mathrm{J}=1.5 \mathrm{~Hz}, 7.3 \mathrm{~Hz}$, aryl $\mathrm{CH}) 7.27(\mathrm{~m}, 2 \mathrm{H}$, aryl CH) $7.07(\mathrm{~m}, 3 \mathrm{H}$, aryl $\mathbf{C H}) 6.95(\mathrm{dd}, 1 \mathrm{H}, \mathrm{J}=17.4 \mathrm{~Hz}, \mathrm{~J}=10.7 \mathrm{~Hz}$, $\left.\mathrm{CH}=\mathrm{CH}_{2}\right) 6.81(\mathrm{dt}, 1 \mathrm{H}, \mathrm{J}=1.5 \mathrm{~Hz}, \mathrm{~J}=7.8 \mathrm{~Hz}$, aryl $\mathrm{CH}) 6.64(\mathrm{~d}, 1 \mathrm{H}, \mathrm{J}=$ $8.3 \mathrm{~Hz}$, aryl CH) $6.34(\mathrm{~d}, 1 \mathrm{H}, \mathrm{J}=8.3 \mathrm{~Hz}$, aryl $\mathrm{CH}) 5.62(\mathrm{dd}, 1 \mathrm{H}, \mathrm{J}=1.7$ $\left.\mathrm{Hz}, 17.4 \mathrm{~Hz}, \mathrm{CH}=\mathrm{CH}_{\mathrm{a}} \mathrm{H}_{\mathrm{b}}\right) 5.25(\mathrm{dd}, 1 \mathrm{H}, \mathrm{J}=1.7 \mathrm{~Hz}, 10.7 \mathrm{~Hz}$, $\left.\mathrm{CH}=\mathrm{CH}_{\mathrm{a}} \mathbf{H}_{\mathbf{b}}\right) 4.89\left(\mathrm{~m}, 2 \mathrm{H}, \mathrm{C}=\mathrm{CH}_{\mathrm{a}} \mathrm{H}_{\mathrm{b}}\right) 4.78\left(\mathrm{~m}, 2 \mathrm{H}, \mathrm{C}=\mathrm{CH}_{\mathbf{a}} \mathbf{H}_{\mathbf{b}}\right) 4.65(\mathrm{~s}, 1 \mathrm{H}, \mathrm{NH}) 2.82(\mathrm{~d}$, $\left.2 \mathrm{H}, \mathrm{J}=13.4 \mathrm{~Hz}, \mathrm{CH}_{\mathbf{a}} \mathrm{H}_{\mathrm{b}}\right) 2.73\left(\mathrm{~d}, 2 \mathrm{H}, \mathrm{J}=13.4 \mathrm{~Hz}, \mathrm{CH}_{\mathrm{a}} \mathbf{H}_{\mathbf{b}}\right) 1.36\left(\mathrm{~s}, 6 \mathrm{H}, \mathrm{CH}_{3}\right) .{ }^{13} \mathrm{C} \mathrm{NMR}$ (125 MHz, $\left.\mathrm{C}_{6} \mathrm{D}_{6}\right): \delta 144.8143 .7143 .1134 .4128 .8127 .9125 .9117 .6116 .9116 .7116 .1$ 61.147 .7 24.8. IR (NEAT, NaCl plates): 3419.0 (m) 3069.9 (m) 2964.4 (s) 2279.8 (s) $1638.8(\mathrm{~m}) 1602.3(\mathrm{~s}) 1577.9(\mathrm{~m}) 1511.2(\mathrm{~s}) 1446.9(\mathrm{~s}) 1374.5(\mathrm{w}) 1261.0(\mathrm{~s}) 1185.9(\mathrm{w})$ 1076.0 (s) 1025.3 (s). HRMS (ESI ${ }^{+}$) Calcd for $\mathrm{C}_{23} \mathrm{H}_{27} \mathrm{~N}$ [M+H]: 318.2216. Found: 318.2217.

4-Methyl-2-(2-methyl-allyl)-2,3-1 H-benzo[b]azepine (7a) [3-Methyl-1-(2-methyl-

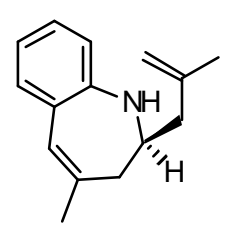
allyl)-but-3-enyl]-(2-vinyl-phenyl)-amine $\mathbf{6 a}(300 \mathrm{mg}, 1.2 \mathrm{mmol})$ and catalyst $5\left[(R) \mathrm{iPr}_{2} \mathrm{TRIP}\right](72 \mathrm{mg}, 62 \mu \mathrm{mol})$ were dissolved in benzene $(15$ $\mathrm{mL}$ ) and heated to $55{ }^{\circ} \mathrm{C}$ under $\mathrm{N}_{2}$ atmosphere for 5 hours. The reaction was cooled, then stirred over charcoal and filtered through celite and washed with ether. Solvents were removed in vacuo to afford a brown solid. This product was purified by column chromatography, eluting with $1 \%$ ether in pentane to afford $215 \mathrm{mg}$ white solid. $\left(240 \mu \mathrm{mol}, 80 \%\right.$ yield) ${ }^{1} \mathrm{H}$ NMR $\left(500 \mathrm{MHz}, \mathrm{C}_{6} \mathrm{D}_{6}\right)$ $\delta 7.12(\mathrm{dd}, 1 \mathrm{H}, \mathrm{J}=1.2 \mathrm{~Hz}, 7.6 \mathrm{~Hz}$, aryl CH) $6.93(\mathrm{dt}, 1 \mathrm{H}, \mathrm{J}=1.5 \mathrm{~Hz}, 7.6 \mathrm{~Hz}$, aryl CH) $6.80(\mathrm{dt}, 1 \mathrm{H}, \mathrm{J}=1.2 \mathrm{~Hz}, 7.3 \mathrm{~Hz}, \operatorname{aryl~CH}) 6.47(\mathrm{~d}, 1 \mathrm{H}, \mathrm{J}=7.6 \mathrm{~Hz}, \operatorname{aryl~CH}) 6.27(\mathrm{~s}, 1 \mathrm{H}$, $\left.\mathrm{ArCH}=\mathrm{CCH}_{3}\right) 4.80\left(\mathrm{~s}, 1 \mathrm{H}, \mathrm{CH}_{3} \mathrm{C}=\mathrm{CH}_{\mathbf{a}} \mathrm{H}_{\mathrm{b}}\right) 4.70\left(\mathrm{~s}, 1 \mathrm{H}, \mathrm{CH}_{3} \mathrm{C}=\mathrm{CH}_{\mathbf{a}} \mathbf{H}_{\mathbf{b}}\right) 3.92(\mathrm{br}, 1 \mathrm{H}, \mathrm{NH})$ $3.18(\mathrm{~m}, 1 \mathrm{H}, \mathrm{NHCH}) 2.31\left(\mathrm{dd}, 1 \mathrm{H}, \mathrm{J}=9.2 \mathrm{~Hz}, 17.4 \mathrm{~Hz}, \mathrm{CH}\left(\mathrm{CH}_{\mathrm{a}} \mathrm{H}_{\mathrm{b}} \mathrm{R}\right)\left(\mathrm{CH}_{2} \mathrm{R}^{\prime}\right)\right) 1.98(\mathrm{~m}$, $\left.3 \mathrm{H}, \mathrm{CH}\left(\mathrm{CH}_{\mathbf{a}} \mathrm{H}_{\mathrm{b}} \mathrm{R}\right)\left(\mathrm{CH}_{2} \mathrm{R}^{\prime}\right)\right) 1.78\left(\mathrm{~s}, 3 \mathrm{H}, \mathrm{CH}_{3}\right) 1.43\left(\mathrm{~s}, 3 \mathrm{H}, \mathrm{CH}_{3}\right) .{ }^{13} \mathrm{C} \mathrm{NMR}(125 \mathrm{MHz}$, $\left.\mathrm{C}_{6} \mathrm{D}_{6}\right) \delta 148.4143 .4136 .1133 .3128 .7127 .5127 .3125 .3120 .1118 .0114 .351 .145 .7$ 45.127 .7 22.0. IR (KBr pellet): 3368.3 (s) $3055.2(\mathrm{~m}) 2881.2(\mathrm{~m}) 1646.4(\mathrm{~m}) 1600.5(\mathrm{~s})$ $1580.1(\mathrm{~m}) 1490.9$ (s) 1442.2 (s) 1283.2 (s) 1199.26 (m) 1050.1 (m). HRMS (EI $\left.{ }^{+}\right)$Calcd for $\mathrm{C}_{17} \mathrm{H}_{23} \mathrm{~N}$ [M+H]: 242.1903. Found: 242.1906. (Chiralcel OJ, $0.1 \% \mathrm{iPrOH}$ in hexane, $1.0 \mathrm{~mL} / \mathrm{min}, 254 \mathrm{~nm}) 93 \%$ e.e. $[\alpha]^{\mathrm{D}}=-22.5 \pm 0.1^{\circ}\left(\mathrm{c}=1.0, \mathrm{CHCl}_{3}\right)$.

2-Ethyl-4-methyl-2-(2-methyl-allyl)- 2,3-dihydro-1 $\boldsymbol{H}$-benzo[ $\boldsymbol{b}]$ azepine (7b) Analogous

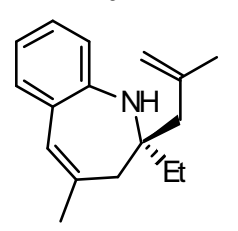
preparation as above, $9.3 \mathrm{mg}$ catalyst $4(9.3 \mu \mathrm{mol})$ and $50 \mathrm{mg} \mathbf{6 b}(186$ $\mu \mathrm{mol})$ used; $32 \mathrm{mg}$ isolated, $132 \mu \mathrm{mol}, 71 \%$ yield. ${ }^{1} \mathrm{H}$ NMR $(500 \mathrm{MHz}$, $\left.\mathrm{C}_{6} \mathrm{D}_{6}\right) \delta 7.07(\mathrm{dd}, 1 \mathrm{H}, \mathrm{J}=1.4 \mathrm{~Hz}, 7.6 \mathrm{~Hz}$, aryl CH) $6.97(\mathrm{dt}, 1 \mathrm{H}, \mathrm{J}=1.5$ $\mathrm{Hz}, 7.6 \mathrm{~Hz}$, aryl CH) $6.77(\mathrm{dt}, 1 \mathrm{H}, \mathrm{J}=1.2 \mathrm{~Hz}, 7.4 \mathrm{~Hz}$, aryl CH) $6.34(\mathrm{dd}$, $1 \mathrm{H}, \mathrm{J}=0.6 \mathrm{~Hz}, 8.1 \mathrm{~Hz}$, aryl CH) $6.30\left(\mathrm{~s}, 1 \mathrm{H}, \mathrm{ArCH}=\mathrm{CR}_{2}\right) 4.88(\mathrm{~m}, 1 \mathrm{H}$, 
$\left.\mathrm{C}=\mathrm{CH}_{\mathrm{a}} \mathrm{H}_{\mathrm{b}}\right) 4.734 .74\left(\mathrm{~m}, 1 \mathrm{H}, \mathrm{C}=\mathrm{CH}_{\mathrm{a}} \mathbf{H}_{\mathrm{b}}\right) 3.81(\mathrm{~s}, 1 \mathrm{H}, \mathrm{NH}) 2.22\left(\mathrm{~m}, 3 \mathrm{H}, \mathrm{CH}_{2} \& \mathrm{CH}_{\mathrm{a}} \mathrm{H}_{\mathrm{b}}\right)$ $2.04\left(\mathrm{~d}, 1 \mathrm{H}, \mathrm{J}=13.6 \mathrm{~Hz}, \mathrm{CH}_{\mathrm{a}} \mathrm{H}_{\mathrm{b}}\right) 1.81\left(\mathrm{~s}, 3 \mathrm{H}, \mathrm{CH}_{3}\right) 1.64\left(\mathrm{~s}, 3 \mathrm{H}, \mathrm{CH}_{3}\right) 1.44(\mathrm{~m}, 2 \mathrm{H}$, $\left.\mathrm{CH}_{2} \mathrm{CH}_{3}\right) 0.72\left(\mathrm{t}, 3 \mathrm{H}, \mathrm{J}=7.5 \mathrm{~Hz}, \mathrm{CH}_{2} \mathrm{CH}_{3}\right) .{ }^{13} \mathrm{C} \mathrm{NMR}\left(125 \mathrm{MHz}, \mathrm{C}_{6} \mathrm{D}_{6}\right) \delta 145.5143 .1$ 133.9133 .1127 .9127 .7124 .4119 .3118 .9116 .0100 .556 .546 .245 .931 .9 28.4 25.2. IR (NEAT, NaCl plate): 3378.8 (m) 3071.2 (m) 2966.7 (s) 1639.9 (m) 1601.9 (s) 1484.8 (s) 1447.5 (m) $1323.5(\mathrm{~m}) 1275.6$ (m) 1160.1 (w) 1059.9 (w). HRMS (EI $\left.{ }^{+}\right)$Calcd for $\mathrm{C}_{17} \mathrm{H}_{23} \mathrm{~N}[\mathrm{M}=\mathrm{H}]:$ 242.1903. Found: 242.1906. (Chiralcel OJ, $1 \% \mathrm{iPrOH}$ in hexane, 1.0 $\mathrm{mL} / \mathrm{min}, 254 \mathrm{~nm}) 24 \%$ e.e.

4-Methyl-2-(2-methyl-allyl)-2-phenyl-2,3-dihydro-1 $H$-benzo[b]azepine

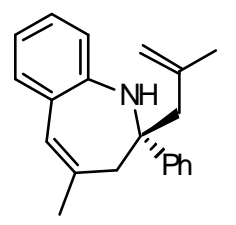

Analogous preparation as above, $10 \mathrm{mg}$ catalyst 4 (10 $\mu \mathrm{mol})$ and $60 \mathrm{mg}$ 6c (189 $\mu \mathrm{mol})$ used; $55 \mathrm{mg}$ isolated, $189 \mu \mathrm{mol}, 99 \%$ yield. ${ }^{1} \mathrm{H}$ NMR (500 $\left.\mathrm{MHz}, \mathrm{C}_{6} \mathrm{D}_{6}\right) \delta 7.18(\mathrm{~m}, 2 \mathrm{H}$, aryl CH) $7.02(\mathrm{~m}, 3 \mathrm{H}$, aryl $\mathrm{CH}) 6.96(\mathrm{~m}, 2 \mathrm{H}$, aryl CH) $7.74(\mathrm{dt}, 1 \mathrm{H}, \mathrm{J}=1.2 \mathrm{~Hz}, 7.4 \mathrm{~Hz}$, aryl $\mathrm{CH}) 6.66(\mathrm{dd}, 1 \mathrm{H}, \mathrm{J}=0.6$ $\mathrm{Hz}, 8.1 \mathrm{~Hz}$, aryl $\mathbf{C H}) 6.08\left(\mathrm{~s}, 1 \mathrm{H}, \operatorname{ArCH}=\mathrm{CR}_{2}\right) 4.84\left(\mathrm{~m}, 1 \mathrm{H}, \mathrm{C}=\mathrm{CH}_{\mathbf{a}} \mathrm{H}_{\mathrm{b}}\right)$ $4.80(\mathrm{~s}, 1 \mathrm{H}, \mathrm{NH}) 4.74\left(\mathrm{~m}, 1 \mathrm{H}, \mathrm{C}=\mathrm{CH}_{\mathrm{a}} \mathbf{H}_{\mathbf{b}}\right) 2.54\left(\mathrm{~s}, 2 \mathrm{H}, \mathrm{CH}_{2}\right) 2.41(\mathrm{~d}, 2 \mathrm{H}, \mathrm{J}=5.5 \mathrm{~Hz}$, $\left.\mathrm{CH}_{2}\right) 1.57\left(\mathrm{~s}, 3 \mathrm{H}, \mathrm{CH}_{3}\right) 1.11\left(\mathrm{~s}, 3 \mathrm{H}, \mathrm{CH}_{3}\right) .{ }^{13} \mathrm{C} \mathrm{NMR}\left(125 \mathrm{MHz}, \mathrm{C}_{6} \mathrm{D}_{6}\right) \delta 146.2145 .6$ 143.2134 .3132 .9128 .7127 .9127 .1126 .7123 .9118 .7118 .4116 .8100 .563 .553 .947 .9 27.8 24.2. IR (NEAT, NaCl plate): 3392.2 (m) 3057.1 (m) 2916.9 (m) 1639.2 (m) 1601.2 (s) 1486.7 (s) 1444.9 (s) 1374.6 (m) 1278.1 (m) 1186.0 (w) 1030.6 (w). HRMS (EI $\left.{ }^{+}\right)$ Calcd for $\mathrm{C}_{21} \mathrm{H}_{23} \mathrm{~N}[\mathrm{M}+\mathrm{H}]: 290.1903$. Found: 290.1905. (Chiralcel OJ, $1 \% \mathrm{iPrOH}$ in hexane, $1.0 \mathrm{~mL} / \mathrm{min}, 254 \mathrm{~nm}) 80 \%$ e.e.

1-Allyl-4-methyl-2-(2-methyl-allyl)-2,3-dihydro-1H-benzo[b]azepine Benzyl

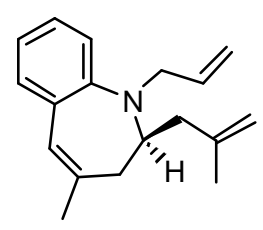
potassium $(80 \mathrm{mg}, 610 \mu \mathrm{mol})$ was added to a solution of 4-Methyl-2-(2methyl-allyl)-2,3-1H-benzo[b]azepine, 7a, (100 mg, $470 \mu \mathrm{mol})$ in THF $(7 \mathrm{~mL})$ at $-35{ }^{\circ} \mathrm{C}$. After warming to room temperature for 35 minutes, allyl bromide ( $74 \mathrm{mg}, 610 \mu \mathrm{mol}$ ) was added to the solution. The reaction was stirred another 2 hours, then quenched on water $(2 \mathrm{~mL})$ and $10 \%$ $\mathrm{HCl}(1 \mathrm{~mL})$. The mixture was extracted three times with ether $(50 \mathrm{~mL})$. All organic extracts were combined, washed with $\mathrm{NaHCO}_{3}, \mathrm{NaCl}$, then dried $\left(\mathrm{MgSO}_{4}\right)$ and concentrated in vacuo. The product was purified by column chromatography, eluting with $1 \%$ ether in pentane to obtain $65 \mathrm{mg}$ of clear, colorless oil. $(260 \mu \mathrm{mol}, 55 \%){ }^{1} \mathrm{H} \mathrm{NMR}$ $\left(500 \mathrm{MHz}, \mathrm{C}_{6} \mathrm{D}_{6}\right) \delta 7.14(\mathrm{dd}, 1 \mathrm{H}, \mathrm{J}=1.5 \mathrm{~Hz}, 7.6 \mathrm{~Hz}$, aryl $\mathrm{CH}) 7.08(\mathrm{dt}, 1 \mathrm{H}, \mathrm{J}=1.8 \mathrm{~Hz}$, $7.6 \mathrm{~Hz}$, aryl CH) $6.87(\mathrm{dt}, 1 \mathrm{H}, \mathrm{J}=1.2 \mathrm{~Hz}, 7.3 \mathrm{~Hz}$, aryl CH) $6.83(\mathrm{~d}, 1 \mathrm{H}, \mathrm{J}=8.2 \mathrm{~Hz}$, aryl CH) $6.37\left(\mathrm{~s}, \quad 1 \mathrm{H}, \quad \operatorname{ArCH}=\mathrm{CCH}_{3}\right) 5.81\left(\mathrm{~m}, 1 \mathrm{H}, \quad \mathrm{CH}_{2} \mathrm{CH}=\mathrm{CH}_{2}\right) 5.13(\mathrm{~m}, 1 \mathrm{H}$, $\left.\mathrm{CH}_{2} \mathrm{CH}=\mathrm{CH}_{\mathrm{a}} \mathrm{H}_{\mathrm{b}}\right) 5.02\left(\mathrm{~m}, 1 \mathrm{H}, \mathrm{CH}_{2} \mathrm{CH}=\mathrm{CH}_{\mathrm{a}} \mathbf{H}_{\mathbf{b}}\right) 4.72\left(\mathrm{~m}, 1 \mathrm{H}, \mathrm{CH}_{3} \mathrm{C}=\mathrm{CH}_{\mathbf{a}} \mathrm{H}_{\mathrm{b}}\right) 4.59(\mathrm{~m}$, $\left.1 \mathrm{H}, \mathrm{CH}_{3} \mathrm{C}=\mathrm{CH}_{\mathrm{a}} \mathbf{H}_{\mathbf{b}}\right) 3.64\left(\mathrm{~m}, 2 \mathrm{H}, \mathrm{NCH}_{2} \mathrm{CH}=\mathrm{CH}_{2}\right) 3.49(\mathrm{~m}, 1 \mathrm{H}, \mathrm{NCH}) 2.27(\mathrm{dd}, 1 \mathrm{H}, \mathrm{J}=$ $\left.1.5 \mathrm{~Hz}, 17.4 \mathrm{~Hz}, \mathrm{CH}\left(\mathrm{CH}_{\mathrm{a}} \mathrm{H}_{\mathrm{b}} \mathrm{R}\right)\left(\mathrm{CH}_{2} \mathrm{R}^{\prime}\right)\right) 2.17(\mathrm{dd}, 1 \mathrm{H}, \mathrm{J}=3.5 \mathrm{~Hz}, 17.4 \mathrm{~Hz}$, $\left.\mathrm{NCH}\left(\mathrm{CH}_{\mathrm{a}} \mathbf{H}_{\mathrm{b}} \mathrm{R}\right)\left(\mathrm{CH}_{2} \mathrm{R}^{\prime}\right)\right) 2.08\left(\mathrm{~m}, 2 \mathrm{H}, \mathrm{NCH}\left(\mathrm{CH}_{2} \mathrm{R}\right)\left(\mathrm{CH}_{2} \mathrm{R}^{\prime}\right)\right) 1.86\left(\mathrm{~s}, 3 \mathrm{H}, \mathrm{CH}_{3}\right) 1.56$ (s, $\left.3 \mathrm{H}, \mathrm{CH}_{3}\right) .{ }^{13} \mathrm{C}$ NMR $\left(125 \mathrm{MHz}, \mathrm{C}_{6} \mathrm{D}_{6}\right) \delta \quad 147.9143 .9137 .1134 .9133 .1129 .2128 .7$ 127.5120 .4120 .3117 .0113 .558 .354 .240 .140 .0 28.0 22.8. IR (NEAT, NaCl plates): 3071.4 (m) 2965.7 (m) 1643.5 (m) 1595.3 (m) 1495.1 (s) 1439.1 (m) 1373.9 (m) 1232.3 (m) $1168.2(\mathrm{~m})$. HRMS $\left(\mathrm{EI}^{+}\right)$Calcd for $\mathrm{C}_{18} \mathrm{H}_{23} \mathrm{~N}$ : 253.1825. Found: 253.1821. [? ] ${ }^{\mathrm{D}}=-0$. $6 \pm 0.1^{\circ}\left(\mathrm{c}=0.3, \mathrm{CHCl}_{3}\right)$. 
6,9-Dimethyl-7,7a,8,11-tetrahydro-11a-aza-dibenzo[a,c]cycloheptene (8) Schrock

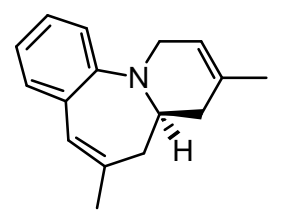

catalyst, 9, $(10 \mathrm{mg}, 13 \mu \mathrm{mol})$ was added to a solution of 1-allyl-4methyl-2-(2-methyl-allyl)-2,3-dihydro- $1 H$-benzo[b]azepine $\quad(65 \mathrm{mg}$, $256 \mu \mathrm{mol})$ in benzene- $d_{6}$. After ten minutes, ${ }^{1} \mathrm{H}$ NMR indicated the reaction was complete. The solution was stirred over charcoal, filtered through celite and concentrated in vacuo. The product was purified by column chromatography eluting with $1 \%$ ether in pentane to afford a white solid, $40 \mathrm{mg}$. $(177 \mu \mathrm{mol}, 69 \%){ }^{1} \mathrm{H}$ NMR $\left(500 \mathrm{MHz}, \mathrm{C}_{6} \mathrm{D}_{6}\right) \delta 7.11(\mathrm{~m}, 2 \mathrm{H}$, aryl $\mathrm{CH}) 6.95(\mathrm{~m}, 2 \mathrm{H}$, aryl CH) $6.37(\mathrm{~s}, 1 \mathrm{H}, \mathrm{ArCH}=\mathrm{C}) 5.24\left(\mathrm{~m}, 1 \mathrm{H}, \mathrm{NCH}_{2} \mathrm{CH}=\mathrm{CCH}_{3}\right) 3.68\left(\mathrm{~m}, 1 \mathrm{H}, \mathrm{NCH}_{\mathrm{a}} \mathrm{H}_{\mathrm{b}} \mathrm{CH}\right)$ $3.49\left(\mathrm{~m}, \quad 1 \mathrm{H} \quad \mathrm{CH}\left(\mathrm{CH}_{2} \mathrm{R}\right)\left(\mathrm{CH}_{2} \mathrm{R}^{\prime}\right)\right) \quad 3.36 \quad\left(\mathrm{~m}, \quad 1 \mathrm{H}, \quad \mathrm{NCH}_{\mathrm{a}} \mathrm{H}_{\mathrm{b}} \mathrm{CH}\right) \quad 2.21 \quad(\mathrm{~m}, \quad 2 \mathrm{H}$, $\left.\mathrm{CH}\left(\mathrm{CH}_{2} \mathrm{R}\right)\left(\mathrm{CH}_{2} \mathrm{R}^{\prime}\right)\right) 1.82$ (s, 3H, $\left.\mathbf{O H}_{3}\right) 1.81$ (m, $\left.2 \mathrm{H}, \mathrm{CH}\left(\mathrm{CH}_{2} \mathrm{R}\right)\left(\mathrm{CH}_{2} \mathrm{R}^{\prime}\right)\right) 1.59$ (s, 3H, $\left.\mathrm{CH}_{3}\right) .{ }^{13} \mathrm{C}$ NMR (125 MHz, $\left.\mathrm{C}_{6} \mathrm{D}_{6}\right) \delta 150.0138 .8133 .2133 .1130 .6128 .7127 .6127 .4$ 121.4120 .9120 .065 .650 .438 .137 .326 .9 23.5. IR (NEAT, NaCl plates): $3060.6(\mathrm{~m})$ $2925.8(\mathrm{~s}) 1593.4(\mathrm{~s}) 1487.4(\mathrm{~s}) 1442.4(\mathrm{~s}) 1386.3(\mathrm{~m}) 1257.0(\mathrm{~m}) 1225.4(\mathrm{~s}) 1178.9(\mathrm{~m})$ $1049.4(\mathrm{~m})$. HRMS $\left(\mathrm{EI}^{+}\right)$Calcd for $\mathrm{C}_{16} \mathrm{H}_{19} \mathrm{~N}$ : 225.1512. Found: 225.1513. HPLC (Chiralcel OJ, $100 \%$ hexane, $0{ }^{\circ} \mathrm{C}, 1.0 \mathrm{~mL} / \mathrm{min}, 254 \mathrm{~nm}$ ) $>90 \%$ e.e. (No baseline.) $[\alpha]^{\mathrm{D}}=$ $+6.5 \pm 0.1^{\circ}\left(\mathrm{c}=1.0, \mathrm{CHCl}_{3}\right)$.

[1,1-Bis-(2-methyl-allyl)-pent-4-enyl]-(2-vinyl-phenyl)-amine (10) Prepared

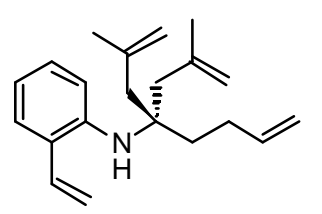
analogously to 1a, $0.64 \mathrm{~g}, 2.2 \mathrm{mmol}, 22 \%$ yield. ${ }^{1} \mathrm{H}$ NMR $(500 \mathrm{MHz}$, $\left.\mathrm{C}_{6} \mathrm{D}_{6}\right): \delta 7.30(\mathrm{dd}, 1 \mathrm{H}, \mathrm{J}=1.5 \mathrm{~Hz}, 7.3 \mathrm{~Hz}$, aryl $\mathrm{CH}) 7.11(\mathrm{dt}, 1 \mathrm{H}, \mathrm{J}=$ $1.8 \mathrm{~Hz}, 7.8 \mathrm{~Hz}$, aryl CH) $6.88(\mathrm{~d}, 1 \mathrm{H}, \mathrm{J}=8.2 \mathrm{~Hz}$, aryl CH) $6.72(\mathrm{~m}$, $2 \mathrm{H}$, aryl $\left.\mathrm{OH}+\mathrm{ArCH}=\mathrm{CH}_{2}\right) 5.73\left(\mathrm{~m}, 1 \mathrm{H}, \mathrm{CH}_{2} \mathrm{CH}_{2} \mathrm{CH}=\mathrm{CH}_{2}\right) 5.49$ $\left(\mathrm{dd}, 1 \mathrm{H}, \mathrm{J}=1.8 \mathrm{~Hz}, 17.1 \mathrm{~Hz}, \mathrm{ArCH}=\mathrm{CH}_{\mathrm{a}} \mathrm{H}_{\mathrm{b}}\right) 5.14(\mathrm{dd}, 1 \mathrm{H}, \mathrm{J}=1.8$ $\left.\mathrm{Hz}, 10.6 \mathrm{~Hz}, \mathrm{ArCH}=\mathrm{CH}_{\mathbf{a}} \mathbf{H}_{\mathbf{b}}\right) 5.01\left(\mathrm{dd}, 1 \mathrm{H}, \mathrm{J}=1.8 \mathrm{~Hz}, 17.1 \mathrm{~Hz}, \mathrm{CH}_{2} \mathrm{CH}_{2} \mathrm{CH}=\mathrm{CH}_{\mathbf{a}} \mathrm{H}_{\mathrm{b}}\right)$ $4.95\left(\mathrm{dd}, 1 \mathrm{H}, \mathrm{J}=1.2 \mathrm{~Hz}, 10.6 \mathrm{~Hz}, \mathrm{CH}_{2} \mathrm{CH}_{2} \mathrm{CH}=\mathrm{CH}_{\mathrm{a}} \mathbf{H}_{\mathbf{b}}\right) 4.89\left(\mathrm{~m}, 2 \mathrm{H}, \mathrm{CH}_{3} \mathrm{C}=\mathrm{CH}_{\mathbf{a}} \mathrm{H}_{\mathrm{b}}\right)$ $4.78\left(\mathrm{~m}, 2 \mathrm{H}, \mathrm{CH}_{3} \mathrm{C}=\mathrm{CH}_{\mathrm{a}} \mathbf{H}_{\mathbf{b}}\right) 3.94(\mathrm{~s}, 1 \mathrm{H}, \mathrm{NH}) 2.47\left(\mathrm{~d}, 2 \mathrm{H}, \mathrm{J}=14.0 \mathrm{~Hz}, \mathrm{C}\left(\mathrm{CH}_{\mathrm{a}} \mathrm{H}_{\mathrm{b}} \mathrm{R}\right)_{2}\right)$ $2.29\left(\mathrm{~d}, 2 \mathrm{H}, \mathrm{J}=14.0 \mathrm{~Hz}, \mathrm{C}\left(\mathrm{CH}_{\mathrm{a}} \mathbf{H}_{\mathbf{b}} \mathrm{R}\right)_{2}\right) 2.07\left(\mathrm{~m}, 2 \mathrm{H}, \mathbf{C H}_{\mathbf{2}}\right) 1.79\left(\mathrm{~m}, 2 \mathrm{H}, \mathbf{C H}_{\mathbf{2}}\right) 1.68(\mathrm{~s}$, 6H, $\left.\mathrm{CH}_{3}\right) .{ }^{13} \mathrm{C}$ NMR (125 MHz, $\left.\mathrm{C}_{6} \mathrm{D}_{6}\right): \delta 144.4143 .0138 .9134 .5129 .4129 .1126 .4$ 117.6117 .0116 .1115 .0114 .058 .944 .737 .7 29.2 25.1. IR (NEAT, NaCl plates): 3414.8 (m) $3074.0(\mathrm{~m}) 29.46 .0(\mathrm{~s}) 1640.2(\mathrm{~s}) 1602.4(\mathrm{~s}) 1578.9(\mathrm{~s}) 1513.9(\mathrm{~s}) 1454.1(\mathrm{~s}) 1373.8$ (m) 1317.5 (s) $1260.0(\mathrm{~m}) 1163.4(\mathrm{~m}) 1058.8(\mathrm{~m})$. HRMS (ESI) Calcd for $\mathrm{C}_{21} \mathrm{H}_{29} \mathrm{~N}[\mathrm{M}+$ H]: 296.2373. Found: 296.2381 .

\section{8,4'-Dimethyl-5,7-dihydrospiro[6H-benzo-5-aza-cycloheptene -6,1'-cyclohex-3'-ene]}

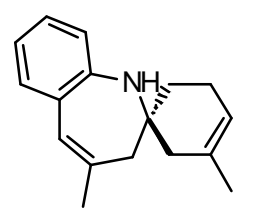

(12) Catalyst $3\left[(R) \mathrm{Pr}_{2} \mathrm{Bnz}_{2} \mathrm{Bitet}\right](20 \mathrm{mg}, 20 \mu \mathrm{mol})$ was added to a solution of $10(110 \mathrm{mg}, 370 \mu \mathrm{mol})$ in benzene $(5 \mathrm{~mL})$, and the resultant mixture placed in a Schlenk flask. The reaction was monitored by ${ }^{1} \mathrm{H}$ NMR analysis. After 1 hour, the ratio of $(\mathbf{1 0}: \mathbf{1 1}: \mathbf{1 2})$ was $(0: 4: 1)$; after 6 hours, only $\mathbf{1 2}$ was observable. The mixture was exposed to air, stirred over charcoal, filtered through celite and silica (eluting with ether in pentane) and concentrated in vacuo to $98 \mathrm{mg}, 360 \mu \mathrm{mol}$, quantitiative yield. ${ }^{1} \mathrm{H} \mathrm{NMR}(500 \mathrm{MHz}$, $\left.\mathrm{C}_{6} \mathrm{D}_{6}\right): \delta 7.08(\mathrm{dd}, 1 \mathrm{H}, \mathrm{J}=1.5 \mathrm{~Hz}, 7.3 \mathrm{~Hz}$, aryl CH) $6.94(\mathrm{dt}, 1 \mathrm{H}, \mathrm{J}=1.5 \mathrm{~Hz}, 7.6 \mathrm{~Hz}$, aryl CH) $6.79(\mathrm{dt}, 1 \mathrm{H}, \mathrm{J}=1.2 \mathrm{~Hz}, 7.3 \mathrm{~Hz}$, aryl CH) $6.35(\mathrm{dd}, 1 \mathrm{H}, \mathrm{J}=1.2 \mathrm{~Hz}, 7.9 \mathrm{~Hz}$, aryl CH) $6.32\left(\mathrm{~m}, 1 \mathrm{H}, \mathrm{ArCH}=\mathrm{CCH}_{3}\right) 5.35\left(\mathrm{~m}, 1 \mathrm{H}, \mathrm{CH}_{2} \mathrm{CH}=\mathrm{CCH}_{3}\right) 3.85(\mathrm{~s}, 1 \mathrm{H}, \mathrm{NH}) 2.43(\mathrm{~d}, 1 \mathrm{H}, \mathrm{J}$ $\left.=17.1 \mathrm{~Hz}, \mathrm{CH}_{\mathbf{a}} \mathrm{H}_{\mathrm{b}}\right) 2.04\left(\mathrm{~d}, 1 \mathrm{H}, \mathrm{J}=17.1 \mathrm{~Hz}, \mathrm{CH}_{\mathbf{a}} \mathbf{H}_{\mathbf{b}}\right) 1.88-1.71\left(\mathrm{~m}, 5 \mathrm{H}, \mathrm{CH}_{2}\right) 1.81(\mathrm{~s}$, 
$\left.3 \mathrm{H}, \mathrm{CH}_{3}\right) 1.52\left(\mathrm{~s}, 3 \mathrm{H}, \mathrm{CH}_{3}\right) 1.24(\mathrm{~m}, 1 \mathrm{H}, \mathrm{CH}) .{ }^{13} \mathrm{C}$ NMR $\left(125 \mathrm{MHz}, \mathrm{C}_{6} \mathrm{D}_{6}\right): \delta 145.7$ 134.0132 .9131 .4128 .7127 .6127 .4125 .3121 .9119 .6119 .552 .249 .043 .930 .428 .4 24.1 23.0. IR (NEAT, NaCl plates): 3368.9 (m) 3051.2 (m) 2911.4 (s) 1601.3 (s) 1482.1 (s) 1446.6 (s) $1376.3(\mathrm{~m}) 1278.0$ (s) 1159.5 (m) 1082.5 (m) 1016.4 (m). HRMS (ESI) Calcd for $\mathrm{C}_{17} \mathrm{H}_{21} \mathrm{~N}[\mathrm{M}+\mathrm{H}$ ]: 240.1747. Found: 240.1756. HPLC: (Chiralcel OJ, $1.0 \%$ Isopropanol in Hexane, $1.0 \mathrm{~mL} / \mathrm{min}, 254 \mathrm{~nm}) 79 \%$ e.e.

\section{4,5-Dihydroxy-4-methyl-2-(2-methyl-allyl)-2,3-1 $H$-benzo[b]azepine}

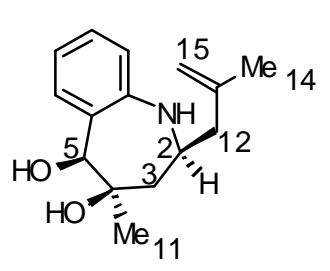
Solid $7 \mathbf{a}(75 \mathrm{mg}, 0.35 \mathrm{mmol})$ was added to a biphasic mixture of AD-mix- $\alpha$ (500 mg, $0.35 \mathrm{mmol}$ ) and methanesulfonamide $(31 \mathrm{mg}$, $0.35 \mathrm{mmol})$ in $t$-butanol/water $(2.5 \mathrm{~mL}$ each $)$ at $0{ }^{\circ} \mathrm{C}$. The mixture was vigorously stirred for 18 hours, then quenched by the addition of sodium sulfite and dichloromethane. The mixture was extracted three times with dichloromethane $(20 \mathrm{~mL})$ dried over $\mathrm{MgSO}_{4}$ and concentrated in vacuo to a brown oil. The crude material was purified via preparative thin layer chromatography on silica gel, eluting with $40 \%$ ethyl acetate in pentane. The clean material $(10 \mathrm{mg}, 39 \mu \mathrm{mol}, 11 \%$ yield $)$ was removed at $\mathrm{R} \sim 0.6 .{ }^{1} \mathrm{H}$ NMR $(500 \mathrm{MHz}$, $\left.\mathrm{C}_{6} \mathrm{D}_{6}\right)$ : ? $7.09(\mathrm{dd}, 1 \mathrm{H}, \mathrm{J}=1.5 \mathrm{~Hz}, 7.3 \mathrm{~Hz}$, aryl CH) $6.98(\mathrm{dt}, 1 \mathrm{H}, \mathrm{J}=1.5 \mathrm{~Hz}, 7.6 \mathrm{~Hz}, \operatorname{aryl}$ CH) $6.81(\mathrm{dt}, 1 \mathrm{H}, \mathrm{J}=1.2 \mathrm{~Hz}, 7.5 \mathrm{~Hz}$, aryl CH) $6.40(\mathrm{dd}, 1 \mathrm{H}, \mathrm{J}=1.2 \mathrm{~Hz}, 7.6 \mathrm{~Hz}$, aryl CH) 4.79 (s, 1H, H15) 4.67 (s, 1H, H15) 4.38 (s, 1H, H5) 4.17 (br, 1H, OH) $3.43(\mathrm{~m}, 1 \mathrm{H}, \mathbf{H 2})$ $3.26(\mathrm{~s}, 1 \mathrm{H}, \mathrm{NH}) 1.76(\mathrm{~m}, 2 \mathrm{H}, \mathbf{H 1 2}) 1.55(\mathrm{dd}, 1 \mathrm{H}, \mathrm{J}=2.7 \mathrm{~Hz}, 14.3 \mathrm{~Hz}, \mathbf{H 3}-$ cis $) 1.44(\mathrm{~s}$, $3 \mathrm{H}, \mathrm{H14}) 1.35$ (dd, $1 \mathrm{H}, \mathrm{J}=11.6 \mathrm{~Hz}, 14.3 \mathrm{~Hz}$, H3-trans) 1.23 (s, 3H, H11). ${ }^{13} \mathrm{C}$ NMR (125 MHz, $\mathrm{C}_{6} \mathrm{D}_{6}$ ): ? 143.3131 .1130 .4121 .7 (C13) 121.1114 .9 (C15) 81.3 (C5) 71.6 (C4) 48.6 (C2) 46.7 (C12) 46.6 (C3) 28.2 (C11) 21.9 (C14). (Assignments based on combination of HSQC, HMBC and nOe which follow.) IR (neat, $\mathrm{NaCl}$ plates): 3584.5 $3397.53071 / 72963.92917 .52849 .1 \quad 1717.61646 .41605 .01374 .4$ 1260.5. Nuclear Overhauser Effect (nOe) spectra are on the following pages. The absolute stereochemistry of the dihydroxylation is based on precendent; ${ }^{4,5}$ assignment of stereochemistry at $\mathrm{C} 2$ based on nOe. There no significant nOe increase for $\mathrm{H}-2$ when Me11 is irradiated. However, based on the nOe arising from irradiation of Me-11 we assigned $1.55 \mathrm{ppm} \mathrm{H}-3$ cis to Me- 11 . When $\mathrm{H}-3_{\text {cis }}$ is irradiated, $\mathrm{H}-2$ experiences a significant nOe, whereas little to no increase is observed for irradiation of $1.22 \mathrm{ppm}, \mathrm{H}$ $3_{\text {trans. }}$. Based on these results, we assign the absolute stereochemistry at C-2 as $(S)$.

(1) Cooper, M. K.; Yaniuk, D. W. J. Organomet. Chem. 1981, 2, 231.

(2) Zessini, J.; Steinbach, J.; Johannsen, B. J. Labelled Compd. Radiopharm. 1999, 42,725 .

(3) Shirakawa, E.; Yamasaki, K.; Hiyama, T. Synthesis 1998, 10, 1544.

(4) Kolb, H. C.; VanNieuwenhze, M. S.; Sharpless, K. B. Chem. Rev. 1994, 94, 2483.

(5) Sharpless, K. B.; Amberg, W.; Bennani, Y. L.; Crispino, G. A.; Hartung, J.; Jeong, K.-S.; Kwong, H.-L.; Morikawa, K.; Wang, Z.-M.; Xu, D.; Zhang, X.-L. J. Org. Chem. 1992, 57, 2768. 
HPLC traces:

2a: Chiralpak AS, 0.1\% IPA in Hexanc, $1.0 \mathrm{~mL} / \mathrm{min}, 25 \%$ nm
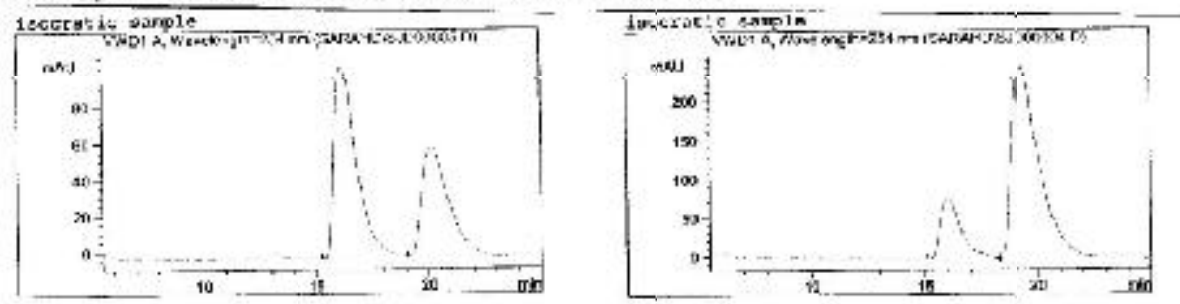

2b: Chiralpak AS, $0.1 \%$ IPA in Hexane, $1.0 \mathrm{~mL} / \mathrm{min}, 254 \mathrm{~nm}$
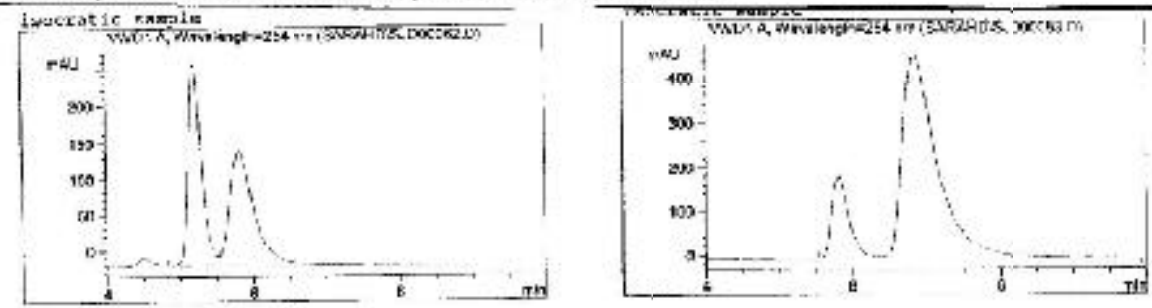

2c: Chiralcel OD, 0\% IPA in Hexane, $1.0 \mathrm{~mL} / \mathrm{min}, 254 \mathrm{rm}$
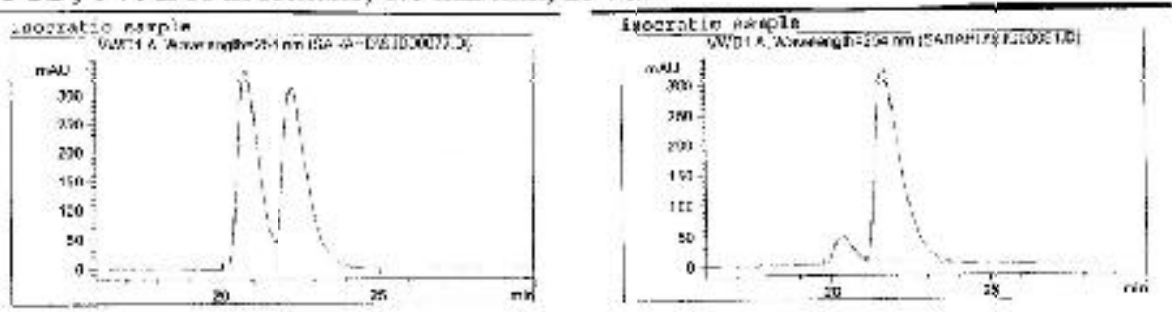

2d: Chiralcel OD, $0.1 \%$ IPA in Hexane, $1.0 \mathrm{~mL} / \mathrm{min}, 25.4 \mathrm{~nm}$
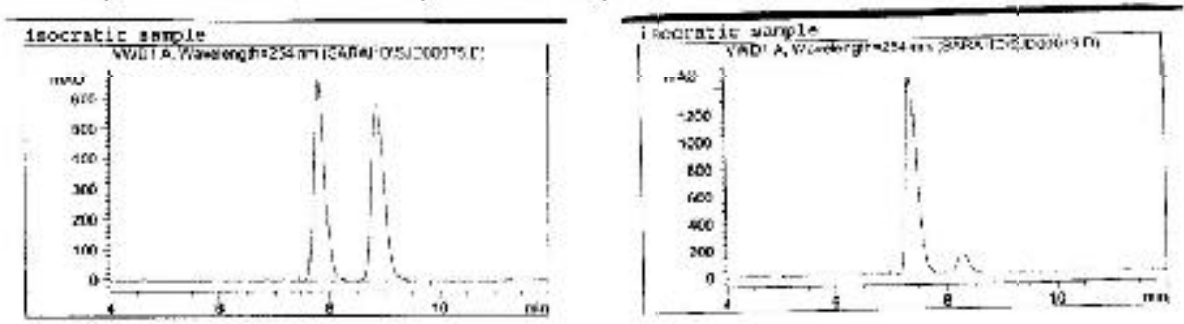

2e: Cuiralcel OD, 0.1\% IPA in Hexanc, $1.0 \mathrm{~mL} / \min , 254 \mathrm{~nm}$
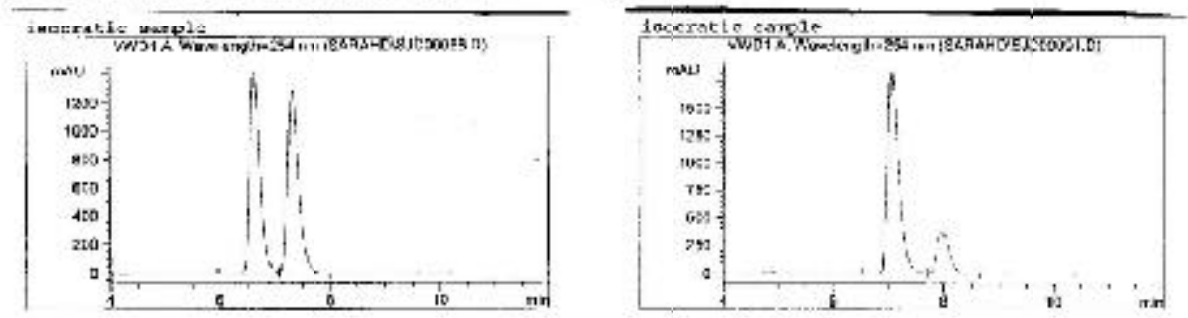
HPLC traces:

7ja: Chiralcel OJ, 0.1\% IPA in Hexane, $1.0 \mathrm{~mL} / \mathrm{min}, 254 \mathrm{~nm}$
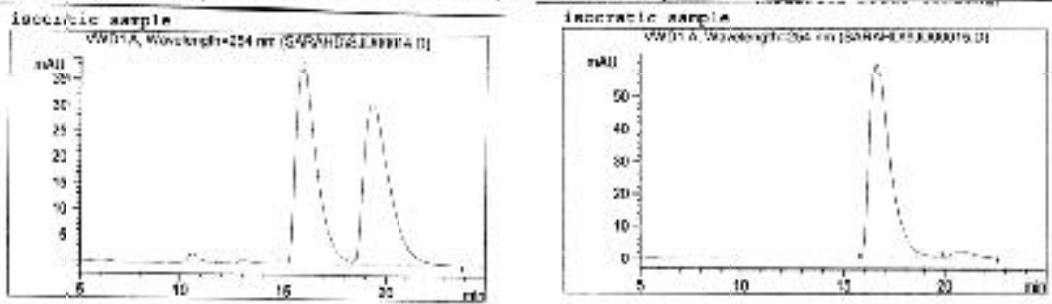

7ib: Ciralcel OJ, 1.0\% IPA in Hexane, $1.0 \mathrm{~mL} / \mathrm{min}, 254 \mathrm{~nm}$
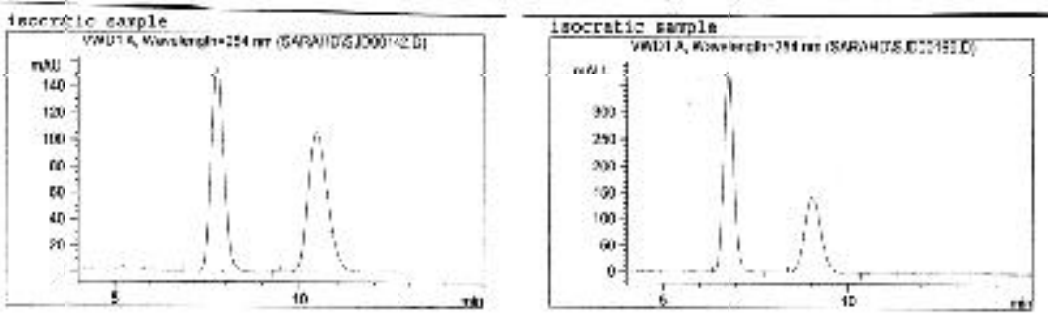

7ic: Chiralcel OJ, $1.0 \%$ IPA in Hexanc, $1.0 \mathrm{~mL} / \mathrm{min}, 254 \mathrm{~nm}$
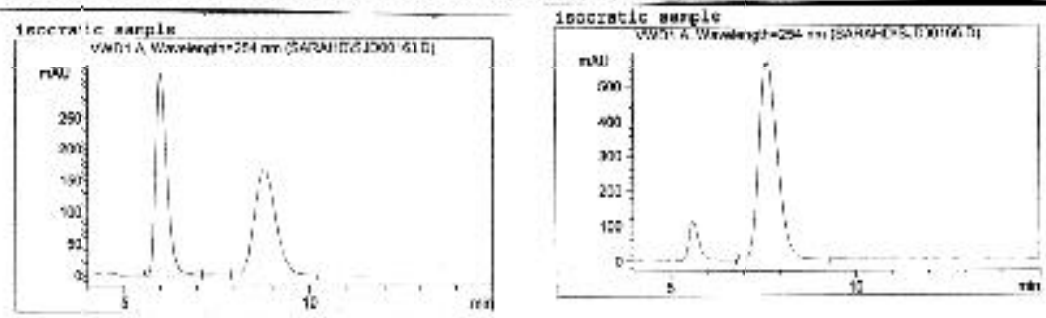

12: Ciralcel OJ, 1.0\% IPA in Hexane, $1.0 \mathrm{~mL} / \mathrm{min}, 254 \mathrm{~nm}$
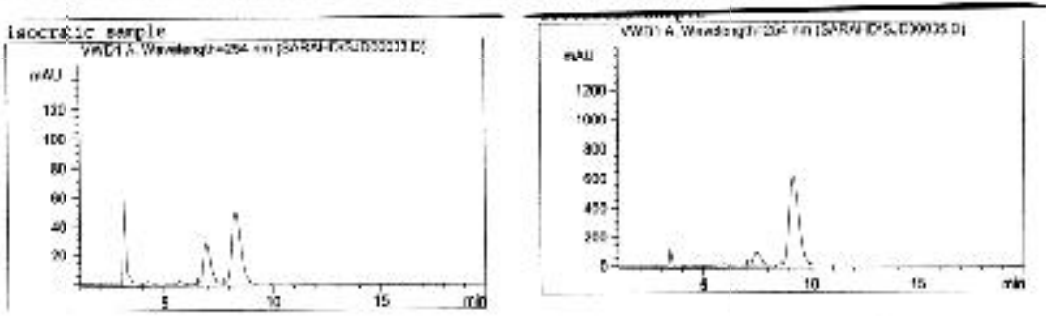
HSQC of 4,5-Dihydroxy-4-methyl-2-(2-methyl-allyl)-2,3-1H-benzo[b]azepine

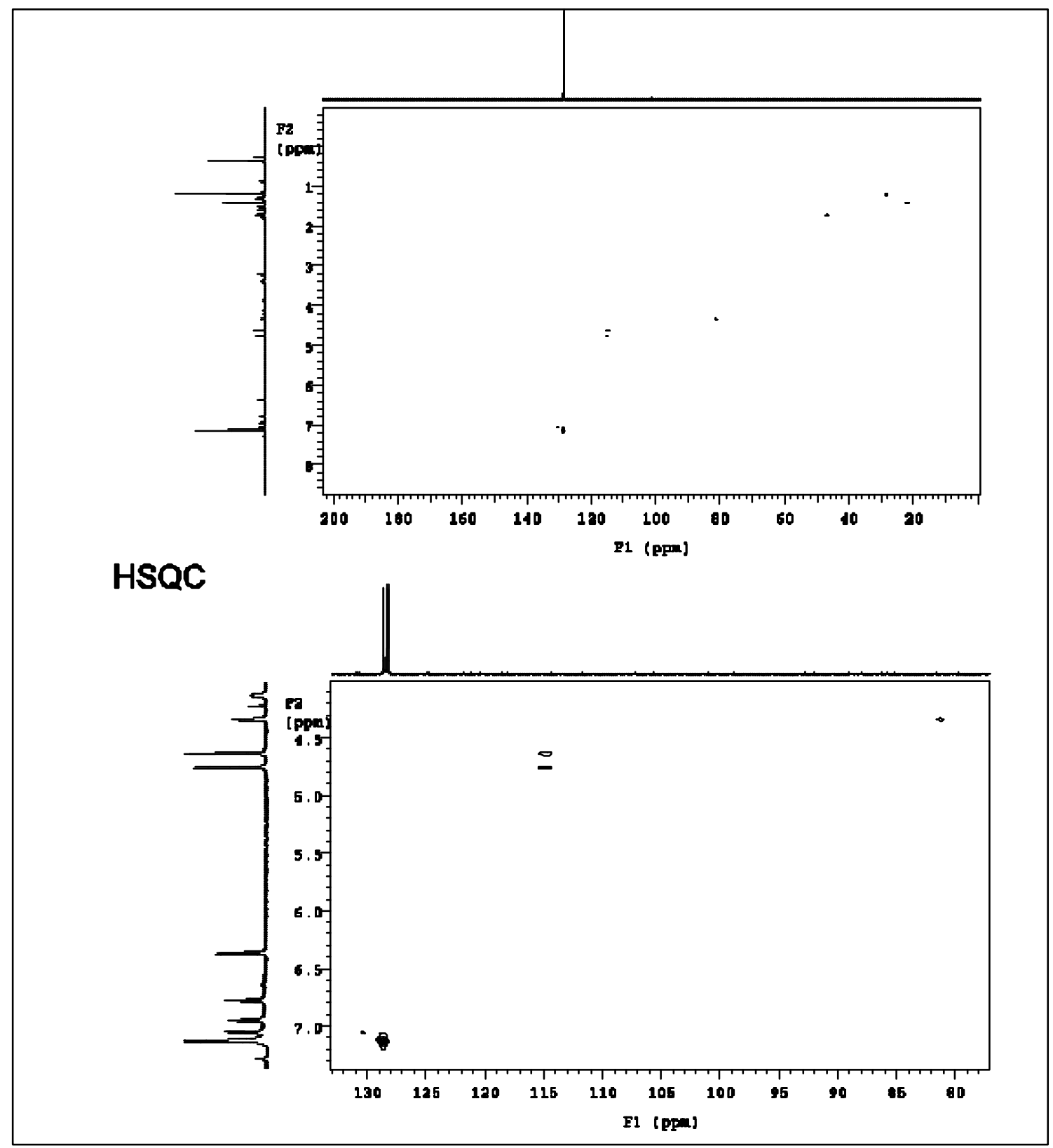




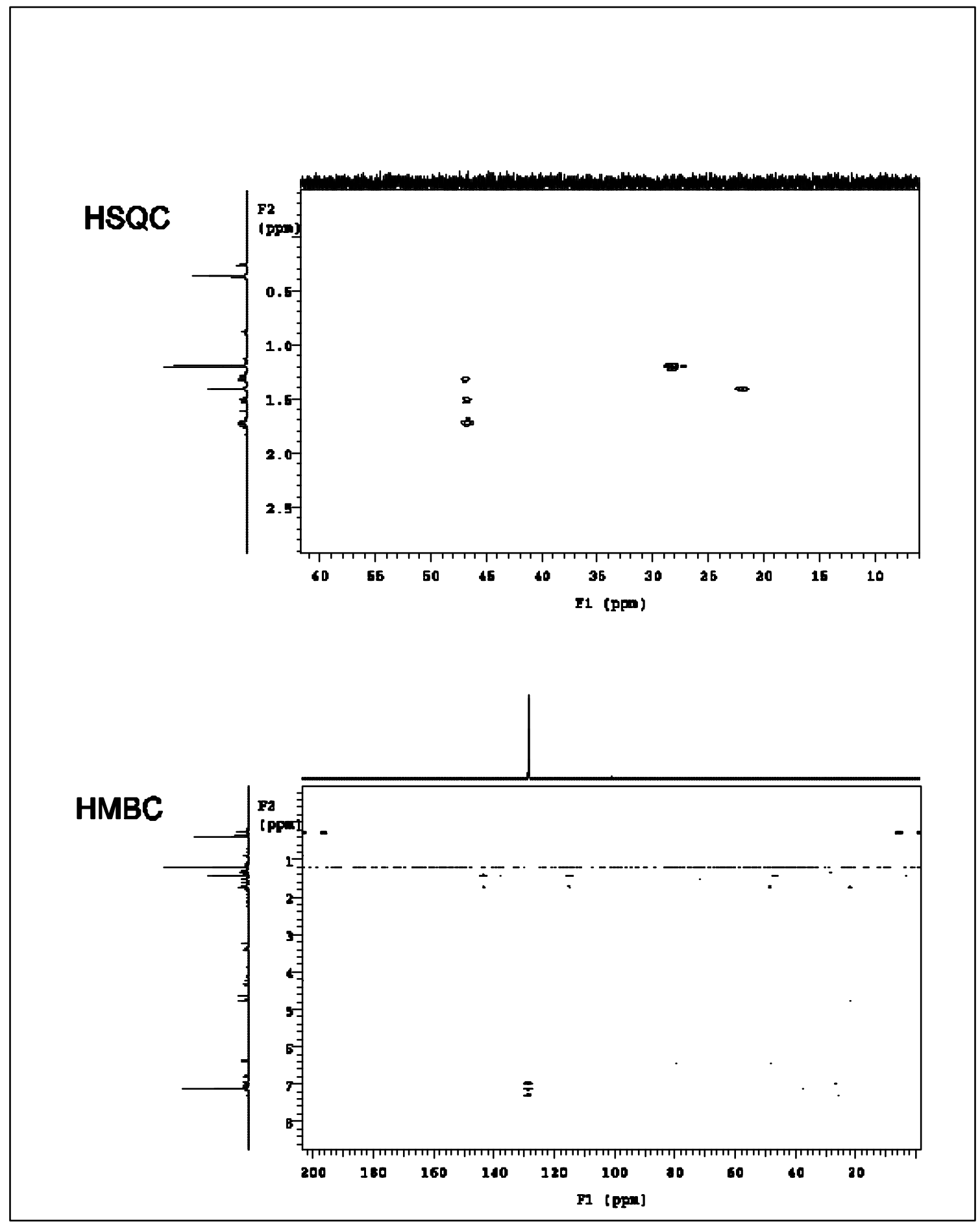




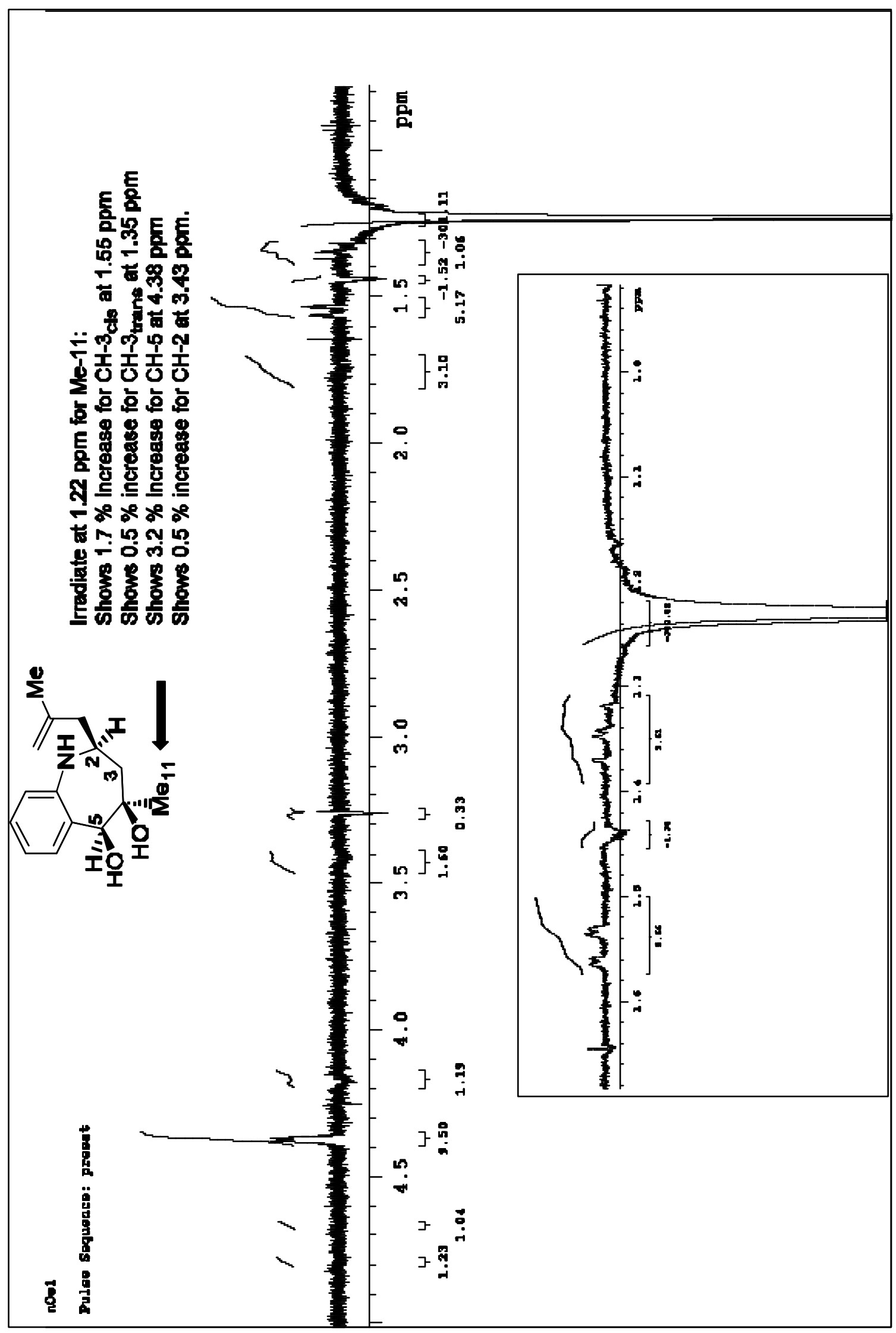




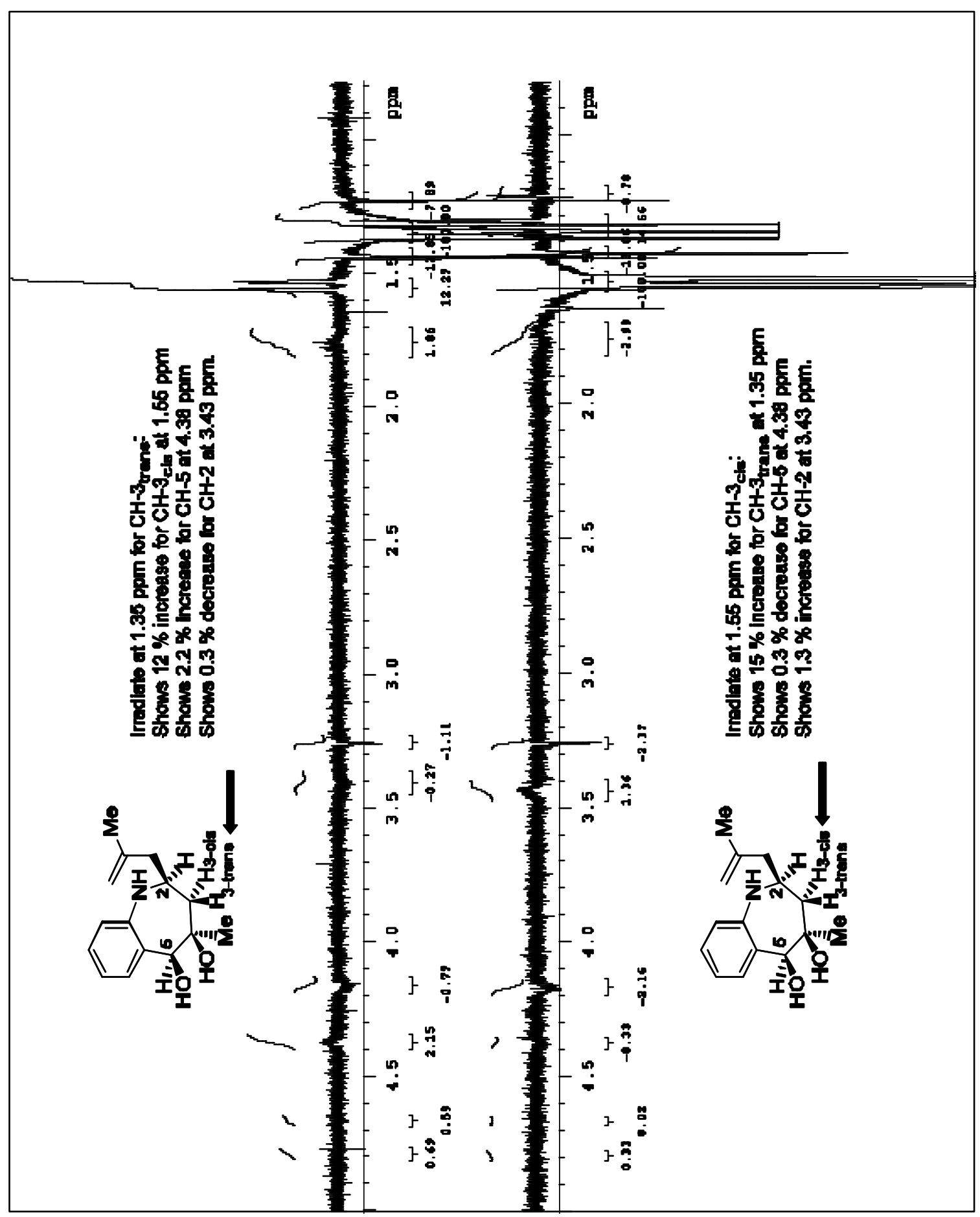

